\title{
Prolate-to-oblate shape phase transitions in neutron-rich odd-mass nuclei
}

\author{
K. Nomura, ${ }^{1}$ R. Rodríguez-Guzmán, ${ }^{2}$ and L. M. Robledo ${ }^{3,4}$ \\ ${ }^{1}$ Physics Department, Faculty of Science, University of Zagreb, HR-10000 Zagreb, Croatia \\ ${ }^{2}$ Physics Department, Kuwait University, 13060 Kuwait, Kuwait \\ ${ }^{3}$ Departamento de Física Teórica, Universidad Autónoma de Madrid, E-28049 Madrid, Spain \\ ${ }^{4}$ Center for Computational Simulation, Universidad Politécnica de Madrid, \\ Campus de Montegancedo, Boadilla del Monte, 28660-Madrid. Spain
}

(Dated: October 16, 2018)

\begin{abstract}
We investigate the prolate-to-oblate shape phase transitions in the neutron-rich $\mathrm{Pt}$, Os and $\mathrm{Ir}$ nuclei in the mass $A \approx 190$ region. The Hamiltonian of the interacting boson-fermion model, used to describe the odd-mass ${ }^{185-199} \mathrm{Pt},{ }^{185-193} \mathrm{Os}$ and ${ }^{185-195} \mathrm{Ir}$ isotopes, is partially constructed by using as a microscopic input the results of constrained self-consistent mean-field calculations within the Hartree-Fock-Bogoliubov method with the Gogny force. The remaining few parameters are adjusted to experimental data in the odd systems. In this way the calculations reasonably describe the spectroscopic properties of the odd-mass systems considered. Several calculated observables for the odd-mass nuclei, especially the low-energy excitation spectra and the effective deformation parameters, point to a prolate-oblate shape transition as a function of the neutron number for all the isotopic chains considered and similar to the one already observed in the neighboring even-even systems.
\end{abstract}

\section{INTRODUCTION}

One of the most prominent features of the atomic nucleus is that it organizes itself into various types of geometrical shapes 1 that often evolve gradually as functions of the nucleon number within an isotopic or isotonic chain. In some instances, however, such shape evolution takes place abruptly at particular nucleon numbers. This phenomenon is known as a (quantum) shape phase transition 22. Over the past decades, numerous experiments have been carried out to measure observables signaling such phase transitions 2. Theoretical calculations have also been carried out within several frameworks [26. Typical examples of shape phase transitions are, the spherical-to-axially-deformed [7] and the spherical-to- $\gamma$ soft [8] ones. Other types of transitions include the one that occurs between prolate and oblate configurations going through a transitional $\gamma$-soft shape [9].

Nuclear shape transitions have been well studied for even-even nuclei. There is, however, a wealth of experimental information for odd-mass systems that remains to be analyzed from a theoretical perspective. Within this context, it is particularly interesting to consider the nature of phase transitions in those odd-mass nuclei and how they correlate with the ones in the neighbouring even-even systems 10. However, the theoretical description of odd-mass nuclei tends to be more cumbersome than for even-even systems, as one needs to take into account both collective and single-particle degrees of freedom on an equal footing [11.

The aim of this paper is to study the effect of the odd particle on the prolate-to-oblate shape transition in neutron-rich nuclei with mass number $A \approx 190$. To this end, we have selected the odd-mass systems ${ }^{185-199} \mathrm{Pt}$ ${ }^{185-193} \mathrm{Os}$ and ${ }^{185-195} \mathrm{Ir}$. Their even-even neighbors ${ }^{186-200} \mathrm{Pt}$ and ${ }^{186-194} \mathrm{Os}$, are considered to be good examples of the prolate-to-oblate shape transition. In many cases $\gamma$-soft shapes are also found. Therefore, they represent a stringent test for nuclear structure models. In order to describe spectroscopic properties, we have resorted to the recently developed method of Ref. [12, based on the nuclear energy density functional (EDF) theory and the particle-core coupling scheme [13, 14]. The method has already been applied to study the spherical-to-axially-deformed [15, 16] and spherical-to- $\gamma$ soft [17, 18] shape phase transitions as well as octupole correlations in neutron-rich Ba nuclei [19. The robustness of the method has been studied using both nonrelativistic [16] and relativistic [17] EDFs.

In this work, the even-even $\mathrm{Pt}$ and Os nuclei are described within the neutron-proton interacting boson model (IBM-2) 20, 21 built on the neutron (proton) $s_{\nu}$ and $d_{\nu}\left(s_{\pi}\right.$ and $\left.d_{\pi}\right)$ bosons, which represent correlated $J^{\pi}=0^{+}$and $2^{+}$pairs of valence neutrons (protons) [20]. On the other hand, the particle-core coupling is considered within the neutron-proton interacting bosonfermion model (IBFM-2) 14, 22. Similar to our previous studies 16, 18, which were based on the simpler IBM-1 model [16], the strength parameters for the IBM-2 Hamiltonian, the single-particle energies and the occupation probabilities of the odd particle, are determined by constrained self-consistent mean-field calculations based on the Gogny-D1M EDF 23, 24. The coupling constants of the boson-fermion interaction are the only free parameters of the model. They are specifically fitted to reproduce the low-lying excitation spectrum for each oddmass nucleus. The IBFM-2 phenomenology has already been considered in this region of the nuclear chart [25]. However, in this work we resort to a microscopic input obtained from the Gogny-D1M EDF framework, i.e., the IBM-2 Hamiltonian parameters, single-particle energies and occupation probabilities are determined within the HFB framework. In a previous study [26, we have already considered even-even nuclei in this mass region, 
including the $\mathrm{Pt}$ and Os ones studied here, with the IBM-2 Hamiltonian parameters derived from HFB calculations based on the Gogny-D1M EDF. In this work, we will take the IBM-2 Hamiltonian parameters for the even-even nuclei from Ref [26] and focus on the remaining ones to study the odd-mass nuclei. Let us also mention that other theoretical frameworks, like the symmetryprojected generator coordinate method (GCM) for odd mass systems [27, 28] and the large-scale shell model [6, 29, could be employed. However, they are computationally much more demanding for heavier and/or openshell nuclei. Hence, computationally feasible schemes, such as the particle-vibration coupling [11, represent a more feasible alternative and have often been considered in the literature, e.g., 30.

The paper is organized as follows. In Sec. [II we briefly outline the theoretical framework used in this study. There, we will also discuss the Gogny-HFB deformation energy surfaces as well as the parameters of the Hamiltonian. Then, in Sec. III, we discuss the spectroscopic properties of the considered nuclei. We briefly review the results obtained for even-even nuclei in Sec. IIA. The systematics of the low-lying yrast levels in the oddmass nuclei is presented in Sec. IIIB, More detailed level schemes and electromagnetic properties for some selected odd-mass nuclei are discussed in Sec. IIIC. As yet another signature of the prolate-to-oblate shape transition, in Sec. IIID, we consider effective $\beta$ and $\gamma$ deformations. Finally, Sec. IV] is devoted to the concluding remarks.

\section{BUILDING THE INTERACTING BOSON-FERMION HAMILTONIAN}

The IBFM-2 Hamiltonian is comprised of the IBM-2 Hamiltonian $\hat{H}_{\mathrm{B}}$ [26], the Hamiltonian for the odd nucleon $\hat{H}_{\mathrm{F}}$, and the boson-fermion interaction $\hat{H}_{\mathrm{BF}}$ :

$$
\hat{H}_{\mathrm{IBFM}}=\hat{H}_{\mathrm{B}}+\hat{H}_{\mathrm{F}}+\hat{H}_{\mathrm{BF}} .
$$

In this expression, the doubly-magic nucleus ${ }^{208} \mathrm{~Pb}$ is taken as the inert core. In the IBM-2, the number of neutron (proton) bosons $N_{\nu}\left(N_{\pi}\right)$ is equal to half the number of valence neutrons (protons) and is counted as the number of holes in the latter half of a given major shell. In the present case, all the bosons are hole-like and therefore $2 \leq N_{\nu} \leq 9$ and $N_{\pi}=2$ for ${ }^{186-200} \mathrm{Pt}$ and $4 \leq N_{\nu} \leq 8$ and $N_{\pi}=3$ for ${ }^{186-194}$ Os. The strength parameters for the IBM-2 Hamiltonian for the even-even nuclei ${ }^{186-200} \mathrm{Pt}$ and ${ }^{186-194}$ Os have been previously determined 31 by mapping the $(\beta, \gamma)$-deformation energy surface, computed within the constrained Gogny-D1M HFB approach, onto the expectation value of the IBM2 Hamiltonian in the boson condensate state 32 . For a more detailed account of the whole procedure, the reader is referred to Refs. 26, 31, 33]. The parameters obtained via the mapping procedure can be found in Table I of Ref. [26].

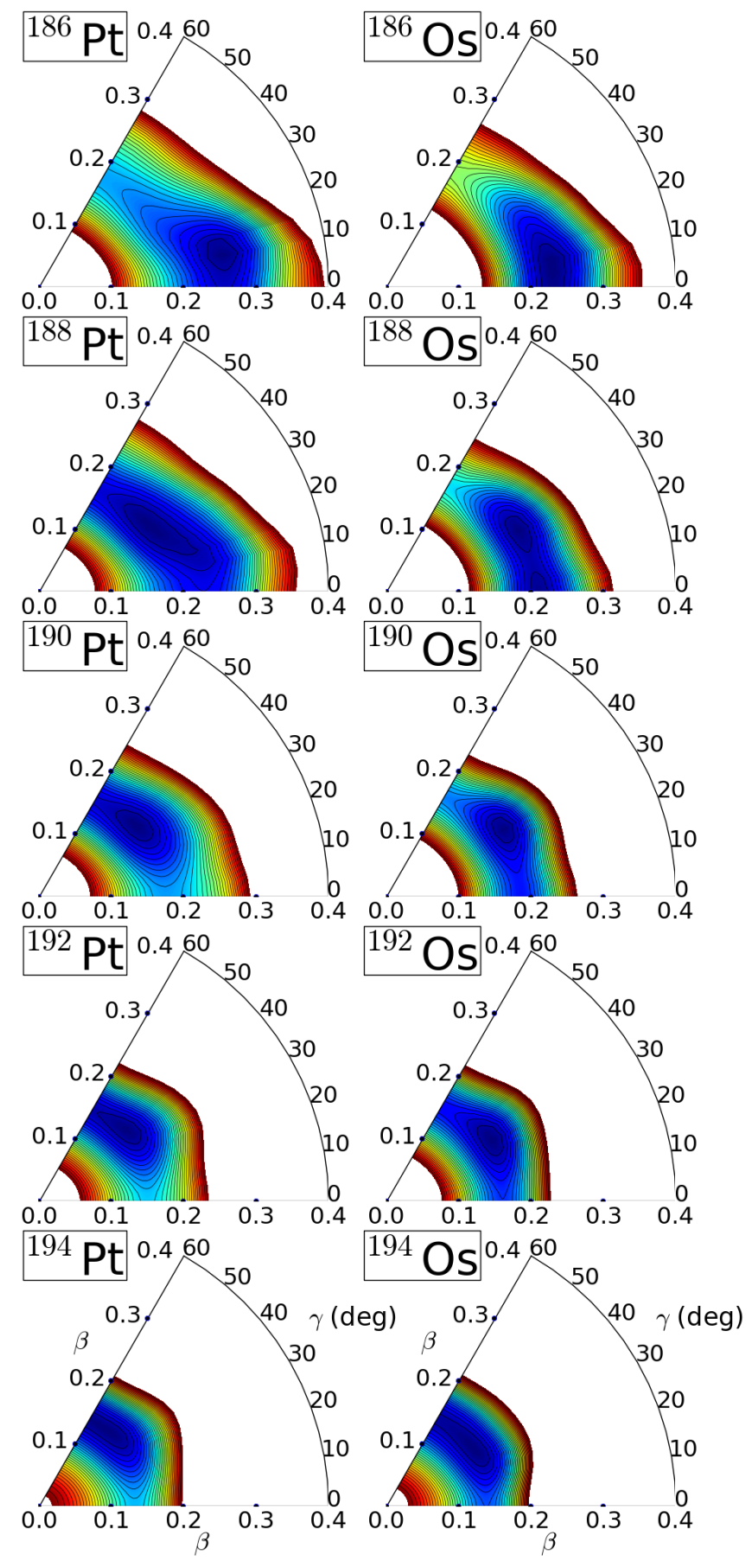

FIG. 1. (Color online) The Gogny-D1M HFB deformation energy surfaces in the $(\beta, \gamma)$-deformation space for the ${ }^{186-194} \mathrm{Pt}$ and ${ }^{186-194}$ Os nuclei are plotted up to $3 \mathrm{MeV}$ from the global minimum. The energy difference between the neighbouring contours is $100 \mathrm{keV}$.

In Fig. 1 we have depicted the Gogny-D1M energy surfaces for those even-even $\mathrm{Pt}$ and Os nuclei corresponding to the prolate-oblate transitional regions. Results for other nuclei can be found in Ref. 4]. Note, that in Ref. 4] we have resorted to the Gogny-D1S 34 EDF. However, 
the mean-field surfaces obtained with the parametrization D1S are essentially the same as the ones provided by the parameter set D1M. The minimum of each of the energy surfaces in the figures changes gradually, as a function of neutron number, from near prolate to shallow triaxial (around ${ }^{188} \mathrm{Pt}$ and ${ }^{190} \mathrm{Os}$ ) and then near oblate (around ${ }^{192} \mathrm{Pt}$ and ${ }^{194} \mathrm{Os}$ ). The IBM-2 energy surfaces, obtained via the mapping procedure, can be found in Ref. 26].

The second term in Eq. 11 reads, $\hat{H}_{\mathrm{F}}=\sum_{j} \epsilon_{j \tau}\left(a_{\tau, j}^{\dagger} \times\right.$ $\left.\tilde{a}_{\tau, j}\right)^{(0)}$, with $\epsilon_{j}$ being the single-particle energies of the orbitals for the odd neutron $(\tau=\nu)$ or proton $(\tau=\pi)$. As fermionic valence space for the odd- $N$ Pt and Os nuclei, we have taken the whole neutron $N=82-126$ major shell: $3 p_{1 / 2}, 3 p_{3 / 2}, 2 f_{5 / 2}, 2 f_{7 / 2}, 1 h_{9 / 2}$ for negative-parity states and $1 i_{13 / 2}$ for positive-parity states. For the odd$Z \mathrm{Ir}$ isotopes we have taken the whole proton $Z=50-82$ major shell: $3 s_{1 / 2}, 2 d_{3 / 2}, 2 d_{5 / 2}, 1 g_{7 / 2}$ for positive parity and $1 h_{11 / 2}$ for negative-parity states. Note that all the valence particles are treated here as holes. Therefore, for an odd-mass nucleus with mass $A$, its even-even neighbor with mass $A+1$ is taken as the even-even boson core.

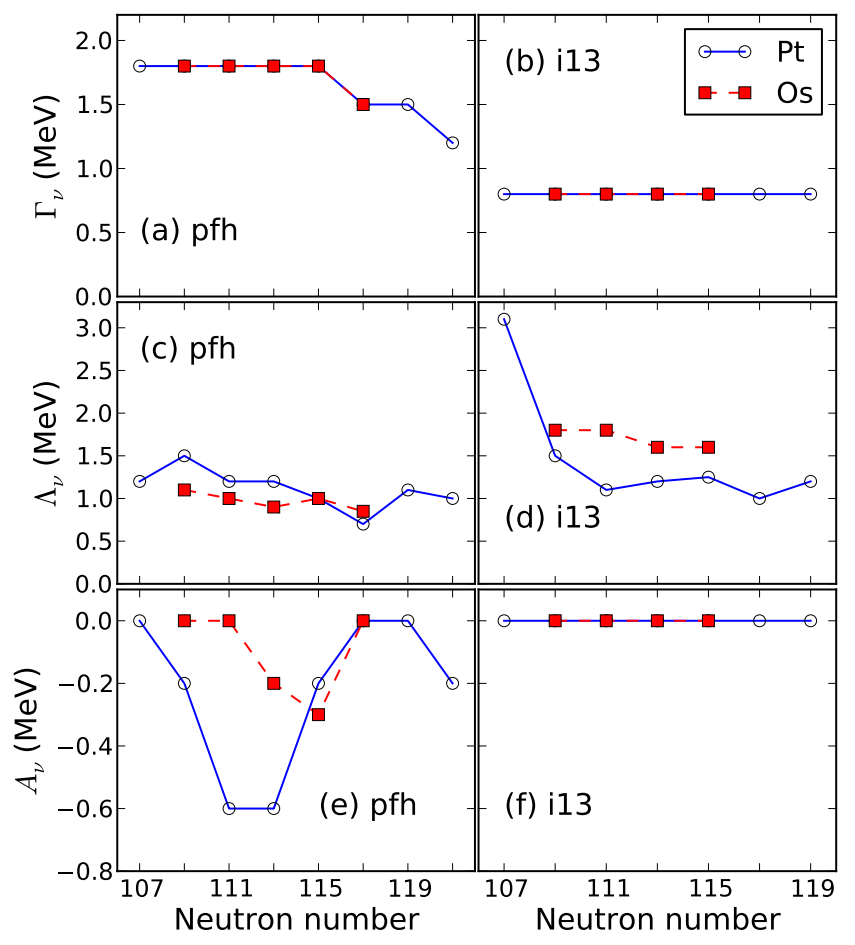

FIG. 2. (Color online) The strength parameters $\Gamma_{\nu}, \Lambda_{\nu}$ and $A_{\nu}$, employed for the odd- $N$ Pt and Os nuclei are depicted as functions of the neutron number.

For the boson-fermion interaction term $\hat{H}_{\mathrm{BF}}$ in Eq. (1), we use an expression similar to the one used in previous studies [25, 35]:

$$
\begin{aligned}
\hat{H}_{\mathrm{BF}}= & \Gamma_{\nu} \hat{Q}_{\pi}^{(2)} \cdot \hat{q}_{\nu}^{(2)}+\Gamma_{\pi} \hat{Q}_{\nu}^{(2)} \cdot \hat{q}_{\pi}^{(2)} \\
& +\Lambda_{\nu} \hat{V}_{\pi \nu}+\Lambda_{\pi} \hat{V}_{\nu \pi}+A_{\nu} \hat{n}_{d \nu} \hat{n}_{\nu}+A_{\pi} \hat{n}_{d \pi} \hat{n}_{\pi}
\end{aligned}
$$

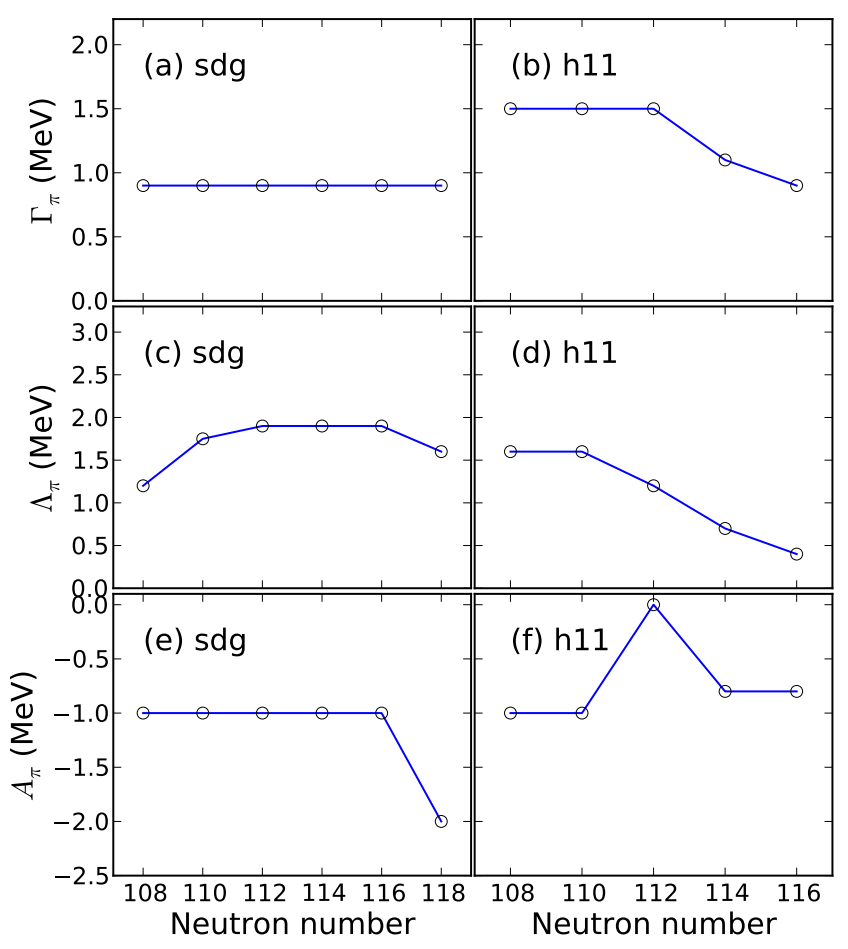

FIG. 3. (Color online) The strength parameters $\Gamma_{\pi}, \Lambda_{\pi}$ and $A_{\pi}$ for the odd- $Z$ Ir isotopes are depicted as functions of the neutron number.

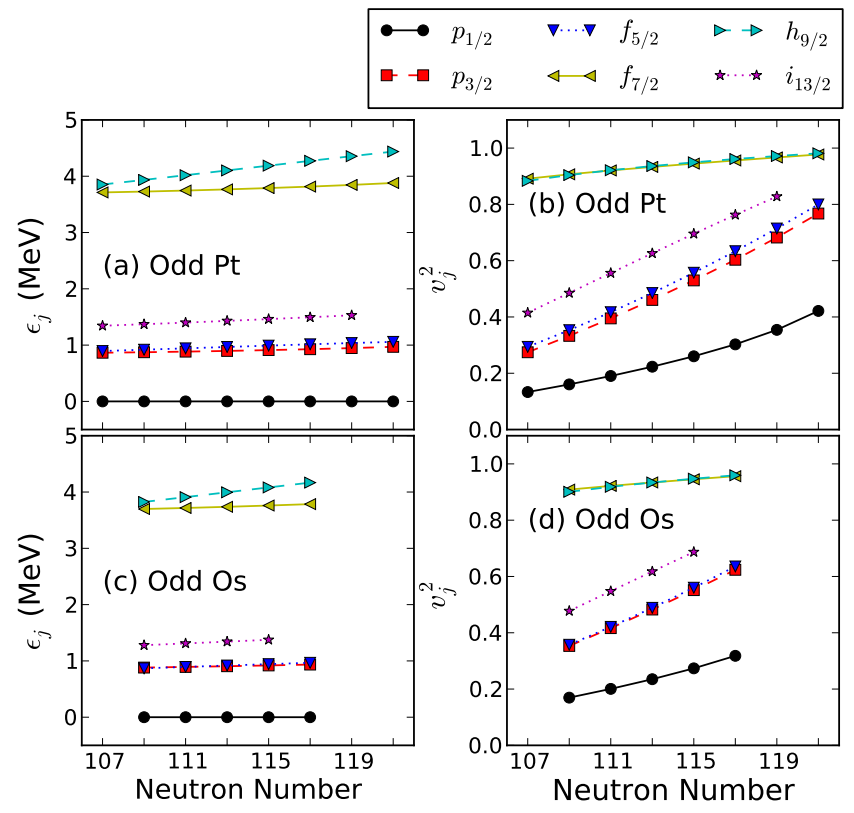

FIG. 4. (Color online) Single-particle energies (plotted with respect to that of the $3 p_{1 / 2}$ orbital) and occupation probabilities employed for the odd- $N$ Pt and Os nuclei as functions of the neutron number.

where the first and second terms are the quadrupole 


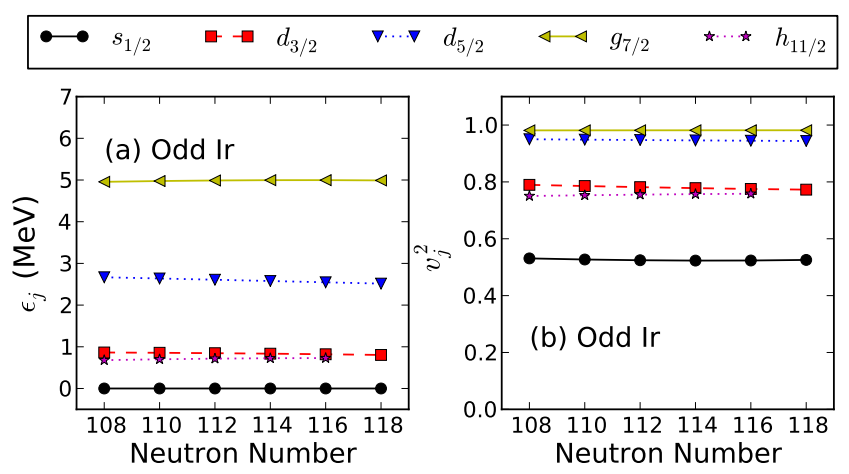

FIG. 5. (Color online) The same as in Fig. 4 but for the odd$Z$ Ir isotopes. The single-particle energies are plotted with respect to $3 s_{1 / 2}$ orbital.

dynamical terms, with the bosonic quadrupole operator for proton $\hat{Q}_{\pi}^{(2)}$ and neutron $\hat{Q}_{\nu}^{(2)}$, respectively. The fermionic quadrupole operator for the odd neutron or proton reads:

$$
\hat{q}_{\tau}^{(2)}=\sum_{j j^{\prime}} \gamma_{j j^{\prime}}\left(a_{j \tau}^{\dagger} \times \tilde{a}_{j^{\prime} \tau}\right)^{(2)}
$$

where $\gamma_{j j^{\prime}}=\left(u_{j} u_{j^{\prime}}-v_{j} v_{j^{\prime}}\right) Q_{j j^{\prime}}$ and $Q_{j j^{\prime}}=$ $\left\langle j\left\|Y^{(2)}\right\| j^{\prime}\right\rangle$ represents the matrix element of the fermionic quadrupole operator in the considered singleparticle basis. The third and fourth terms in Eq. (2) are the exchange interactions. They are introduced to account for the fact that bosons are built from nucleon pairs and are given by [22, 25]

$$
\begin{aligned}
\hat{V}_{\pi \nu}= & -\left(s_{\pi}^{\dagger} \tilde{d}_{\pi}\right)^{(2)} \cdot\left\{\sum_{j j^{\prime} j^{\prime \prime}} \sqrt{\frac{10}{N_{\nu}(2 j+1)}} \beta_{j j^{\prime}} \beta_{j^{\prime \prime} j}\right. \\
& \left.:\left(\left(d_{\nu}^{\dagger} \times \tilde{a}_{j^{\prime \prime} \nu}\right)^{(j)} \times\left(a_{j \nu}^{\dagger} \times \tilde{s}_{\nu}\right)^{\left(j^{\prime}\right)}\right)^{(2)}:\right\}+(\text { H.c. }),
\end{aligned}
$$

with a similar expression for $\hat{V}_{\nu \pi}$. In Eq. (4), $\beta_{j j^{\prime}}=$ $\left(u_{j} v_{j^{\prime}}+v_{j} u_{j^{\prime}}\right) Q_{j j^{\prime}}$. The fifth and sixth terms in Eq. (2) are the monopole interactions with $\hat{n}_{d \nu}$ and $\hat{n}_{d \pi}$ the number operators for neutron and proton $d$ bosons, respectively, while the number operator for the odd fermion $\hat{n}_{\tau}=\sum_{j}(-\sqrt{2 j+1})\left(a_{j \tau}^{\dagger} \times \tilde{a}_{j \tau}\right)^{(0)}$.

The boson-fermion Hamiltonian $\hat{H}_{\mathrm{BF}}$ in Eq. (2) has been justified from microscopic considerations based on the generalized seniority scheme [14, 35]. Both the quadrupole dynamic and exchange terms act predominantly between protons and neutrons (i.e., between odd neutron and proton bosons and between odd proton and neutron bosons) [22, while the monopole interaction acts between like-particles (i.e., between odd neutron and neutron bosons and between odd proton and proton bosons) [14.
The single-particle energies $\epsilon_{j \tau}$ and occupation probabilities $v_{j}^{2}$ of the odd nucleon at the $j$ orbital are obtained from Gogny-D1M HFB calculations constrained to quadrupole moment zero. Note that this is a standard HFB calculation without blocking. However, the particle number is constrained to odd $N$ or $Z$. For more details, see Ref. [16].

The coupling constants $\Gamma_{\nu}, \Gamma_{\pi}, \Lambda_{\nu}, \Lambda_{\pi}, A_{\nu}$, and $A_{\pi}$ in Eq. (2) are treated as free parameters. They have been fitted to reproduce the energies of the lowest-lying states in each of the odd-mass nuclei, separately for normalparity and unique-parity configurations. Those parameters have been plotted in Figs. 2 (odd- $N$ Pt and Os nuclei) and 3 (odd- $Z$ Ir nuclei). As can be seen in Figs.2(a) and $2(\mathrm{~b})$, the $\Gamma_{\nu}$ values for both the normal-parity (denoted by pfh) and unique-parity (i13) configurations stay rather constant as a function of neutron number. The $\Lambda_{\nu}$ values, in Figs. 2(c) and 2(d) also look rather insensitive to variations in neutron number except for the abrupt change in the $\Lambda_{\nu}$ value for the $i_{13 / 2}$ configuration (see, Fig. 2(d)) in going from ${ }^{185} \mathrm{Pt}$ to ${ }^{187} \mathrm{Pt}$. As shown later in Fig. 8, the abrupt change observed is very likely a consequence of the evolution of the low-lying positive-parity level structure when going from one nucleus to the other. Furthermore, as can be seen from Figs.2(e) and 2(f), the monopole strength $A_{\nu}$ is chosen to be zero in many of the studied nuclei. Nevertheless, a relatively large value is needed for the transitional regions, i.e., ${ }^{189,191} \mathrm{Pt}$ and ${ }^{189,191}$ Os. The $\Gamma_{\pi}, \Lambda_{\pi}$ and $A_{\pi}$ values for the odd- $Z$ Ir isotopes are shown in Fig. 3 . They are also nearly constant or change only moderately as functions of the neutron number $N$. Since the monopole interaction turns out to play a major role for most of the considered Ir nuclei, its strength parameter is much larger in magnitude than in the case of the odd-mass Pt and Os nuclei.

In addition, we plot in Figs. 4 and 5 the single-particle energies and occupation probabilities used in the present study for the considered odd-mass nuclei.

The IBFM Hamiltonian of Eq. (1), with parameters determined via the mapping procedure, has been diagonalized to obtain excitation spectra and electromagnetic transition rates. For the later, E2 and M1 operators similar to those in Refs [16, 18, have been used. The effective bosonic charge has been taken to be the same for both protons and neutrons, with a value $e_{\mathrm{B}}^{\nu}=e_{\mathrm{B}}^{\pi}=0.15 e \mathrm{~b}$ fitted to reproduce the experimental $B\left(E 2 ; 2_{1}^{+} \rightarrow 0_{1}^{+}\right)$transition rate in ${ }^{196} \mathrm{Pt}$. For the fermion effective charges we have used the values $e_{\mathrm{F}}^{\nu}=0.3 \mathrm{eb}$ and $e_{\mathrm{F}}^{\pi}=0.5 \mathrm{eb}$. Moreover, for the bosonic $g$-factor we have also taken the same value for protons and neutrons $g_{\mathrm{B}}^{\nu}=g_{\mathrm{B}}^{\pi}=0.3 \mu_{N}$ that is fitted to reproduce the magnetic moment of the $2_{1}^{+}$state in ${ }^{196} \mathrm{Pt}$. For the fermionic $g$-factors, we have adopted $g_{l}=1.0 \mu_{N}$ for the odd proton and $g_{l}=0 \mu_{N}$ for the odd neutron. The free values of $g_{s}$ have been quenched by 30 $\%$. 


\section{RESULTS FOR SPECTROSCOPIC PROPERTIES}

In this section, we discuss the results of this study. First, in Sec. IIIA, we briefly discuss the results obtained for even-even nuclei. The systematics of the low-lying yrast levels in the odd-mass nuclei is presented in Sec. IIIB A more detailed analysis of the level schemes and electromagnetic properties for some selected odd-mass nuclei is presented in Sec. IIIC. Finally, in Sec. IIID we consider effective $\beta$ and $\gamma$ deformation parameters as another signature of the prolate-to-oblate shape transition.

\section{A. Even-even nuclei}

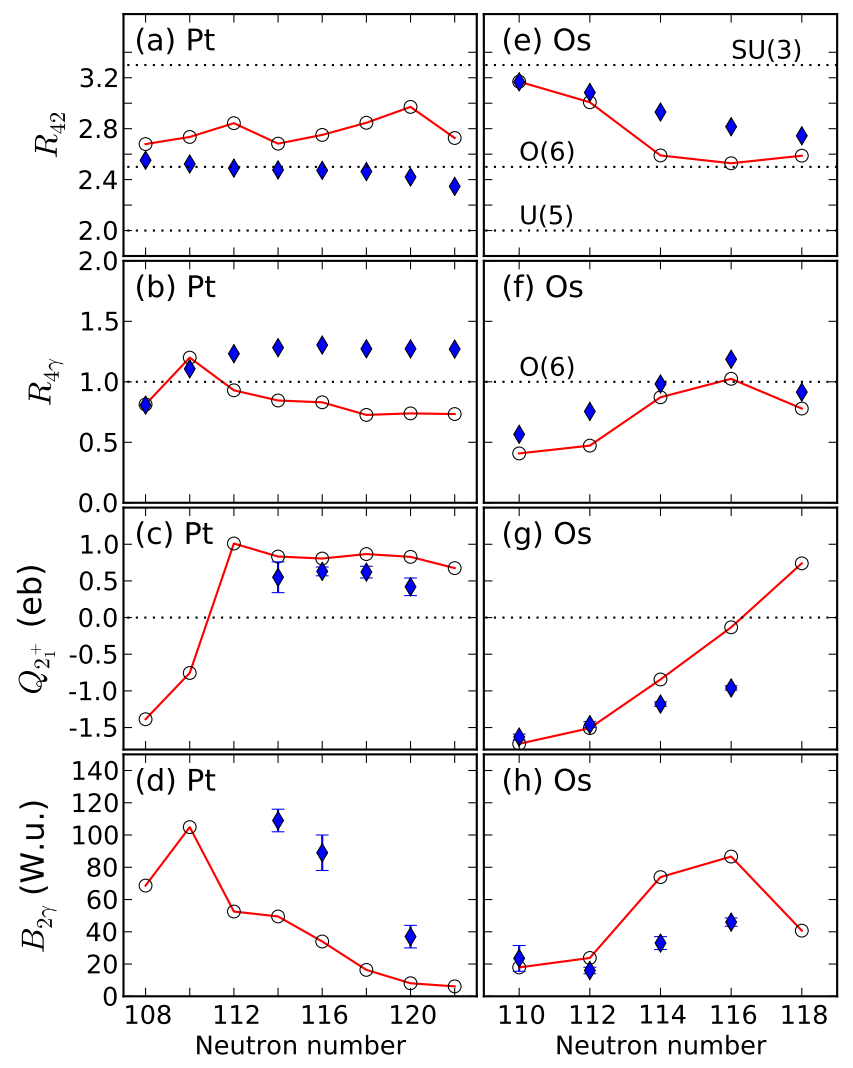

FIG. 6. (Color online) Spectroscopic properties of the eveneven ${ }^{186-200} \mathrm{Pt}$ and ${ }^{186-194}$ Os nuclei plotted as functions of the neutron number: the energy ratios $R_{42}$ and $R_{4 \gamma}$, spectroscopic quadrupole moment $Q_{2_{1}^{+}}$and the $B(E 2)$ value $B_{2 \gamma}$. For more details, see the main text. Open circles, connected by lines, represent the theoretical values while solid diamonds represent the experimental data taken from Refs. 36, 37. The symmetry limits $R_{42}=2.0(\mathrm{U}(5)), 2.5(\mathrm{O}(6))$ and $3.3(\mathrm{SU}(3))$, and $R_{4 \gamma}=1.0(\mathrm{O}(6))$ of the IBM [21] are also indicated.

Let us first consider how the IBM-2 Hamiltonian, extracted from the Gogny-D1M HFB calculations via the mapping procedure, describes the spectroscopic properties of the even-even nuclei. To this end, in Fig. 6, we have plotted spectroscopic properties of ${ }^{186-200} \mathrm{Pt}$ and ${ }^{186-194} \mathrm{Os}$ as functions of the neutron number $N$. The symmetry limits $R_{42}=2.0(\mathrm{U}(5)), 2.5(\mathrm{O}(6))$ and 3.3 $(\mathrm{SU}(3))$ and $R_{4 \gamma}=1.0(\mathrm{O}(6))$ of the IBM [21, are also indicated in the figure. As can be seen in Fig. 6(a), both the theoretical and experimental $R_{42}=E\left(4_{1}^{+}\right) / E\left(2_{1}^{+}\right)$ ratios for $\mathrm{Pt}$ isotopes do not change too much and are located around the $\mathrm{O}(6)$ limit $R_{42} \approx 2.5$. On the other hand, from Fig. 6(a), one observes that the theoretical ratio $R_{42}$ systematically overestimates the experimental one for the heavier isotopes. This might be a consequence of the pronounced ground state deformation in the corresponding Gogny-D1M energy surfaces when approaching the neutron closed shell $N=126$. As a result, the IBM2 model provides a more rotational-like spectrum. The $R_{42}$, displayed in Fig. 6 for Os isotopes, changes rather fast with $N$ compared to the Pt isotopes. The energy ratio $R_{4 \gamma}=E\left(4_{1}^{+}\right) / E\left(2_{2}^{+}\right)$, depicted in Figs. 6(b) and $6(f)$, can be regarded as a signature of $\gamma$-softness. The predicted $R_{4 \gamma}$ values for both Pt and Os nuclei exhibit a peak at around ${ }^{188} \mathrm{Pt}(N=110)$ and ${ }^{192} \mathrm{Os}(N=116)$, being close to the $\mathrm{O}(6)$ limit of $R_{4 \gamma}=1.0$. This indicates that those nuclei represent the most $\gamma$-soft among the considered systems. Indeed, the Gogny-D1M energy surface for ${ }^{188} \mathrm{Pt}$ exhibits the most pronounced triaxial minimum at $\gamma \approx 30^{\circ}$ (see, Fig. 1). As can be seen from Fig. 6(b), both theoretically and experimentally, the ratio $R_{4 \gamma}$ remains rather constant in the case of $\mathrm{Pt}$ isotopes. However, the experimental $R_{4 \gamma}$ values are systematically underestimated for $N \geq 112$. This may be due to a similar reason as with the discrepancy in $R_{42}$ already mentioned.

The spectroscopic quadrupole moment $Q_{2_{1}^{+}}$for the $2_{1}^{+}$ state, displayed in Figs. 6.(c) and 6(g), represents a useful measure of whether the nucleus is prolate or oblate. As can be seen in Fig. 6(c), the theoretical $Q_{2_{1}^{+}}$value is negative for ${ }^{186,188} \mathrm{Pt}$ while it is positive for ${ }^{190-200} \mathrm{Pt}$. This is consistent with the prolate-to-oblate shape transition observed at the mean-field level in Fig. 1. A similar observation can be made for the Os isotopes in Fig. 6(g). Furthermore, the $B_{2 \gamma}=B\left(E 2 ; 2_{2}^{+} \rightarrow 2_{1}^{+}\right)$transition probability provides a stringent test for $\gamma$-softness. For both $\mathrm{Pt}$ and Os isotopes, it shows a peak at $N=110$ (Fig. 6(d)) and 116 (Fig. 6(h)).

\section{B. Systematics of low-energy excitation spectra in odd-mass nuclei}

In Figs. 7 to 12 , we have plotted the energy systematics of the low-lying positive- and negative-parity yrast states in the odd- $N$ nuclei ${ }^{185-199} \mathrm{Pt},{ }^{185-193} \mathrm{Os}$ and ${ }^{185-195} \mathrm{Ir}$ as functions of $N$. For the three isotopic chains, our calculations describe reasonably well the experimental trend. For the odd- $N$ Pt isotopes, in Figs. 7 and 8 , the observed change in the ground state's spin in going from 


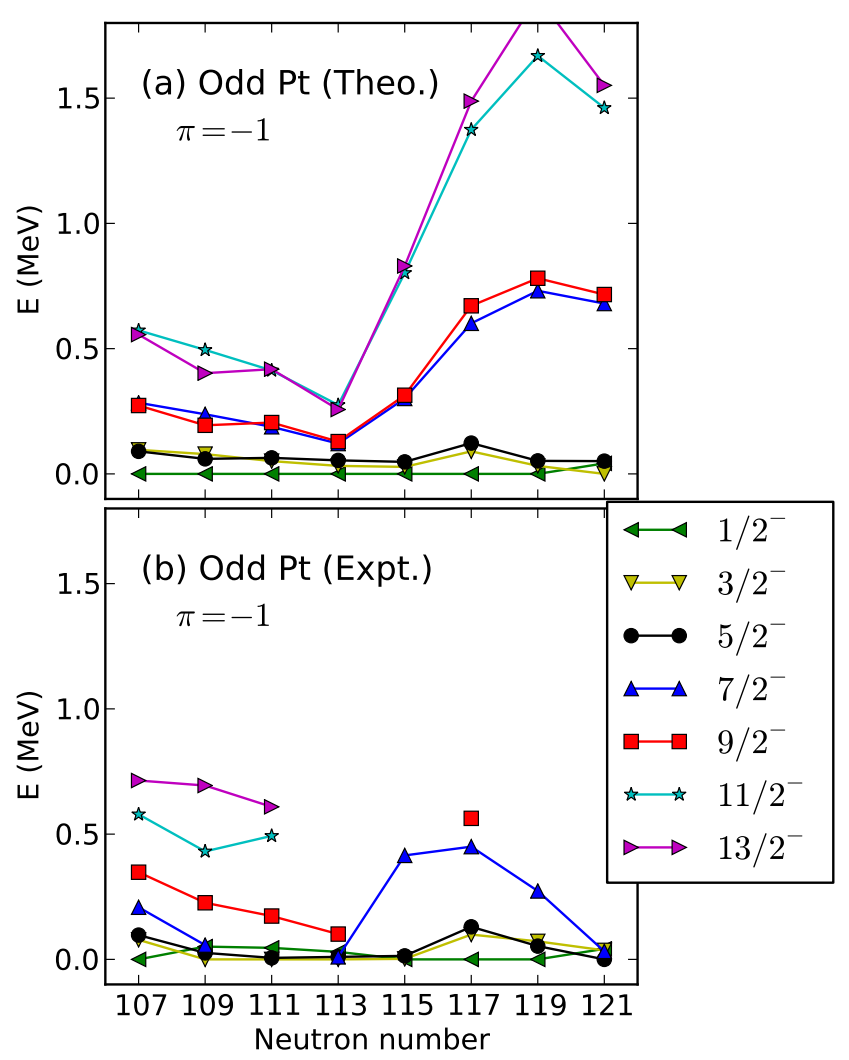

FIG. 7. (Color online) The theoretical and experimental lowlying negative-parity $(\pi=-1)$ yrast states in the odd- $N$ isotopes ${ }^{185-199} \mathrm{Pt}$ are plotted as functions of the neutron number.

$N=107$ to 109 for both parities, can be regarded as a signature of structural evolution and correlates well with the shape transition that occurs in the corresponding eveneven systems. Indeed, the Gogny-D1M energy surfaces (see, Fig. 1) suggest the transition from prolate $\left({ }^{186} \mathrm{Pt}\right)$ to triaxial shapes $\left({ }^{188} \mathrm{Pt}\right)$. For $N=109-113$, both theoretically and experimentally as well as for both parities, a similar low-lying level structure is observed. However, as seen from Fig. 7(a), another signature of the shape transition appears in the case of the negative-parity states for odd- $N$ Pt isotopes, i.e., at the neutron number $N=113$ many states are found below $0.3 \mathrm{MeV}$ excitation energy while those levels higher than the $J=5 / 2^{-}$one go up rapidly for larger $N$. This also correlates well with the Gogny-D1M energy surfaces obtained for even-even nuclei (see, Fig. 1) which exhibit a gradual change of the global minimum from shallow triaxial $\left({ }^{192} \mathrm{Pt}\right)$ to oblate $\left({ }^{194} \mathrm{Pt}\right)$.

The results obtained for odd- $N$ Os isotopes are shown in Figs. 9 and 10 . For the negative-parity states, in Fig. 9 . both experimentally and theoretically the low-lying level structure below $0.3 \mathrm{MeV}$ excitation energy changes significantly around $N=115$, including a change in the ground state's spin. Once more, this agrees well with

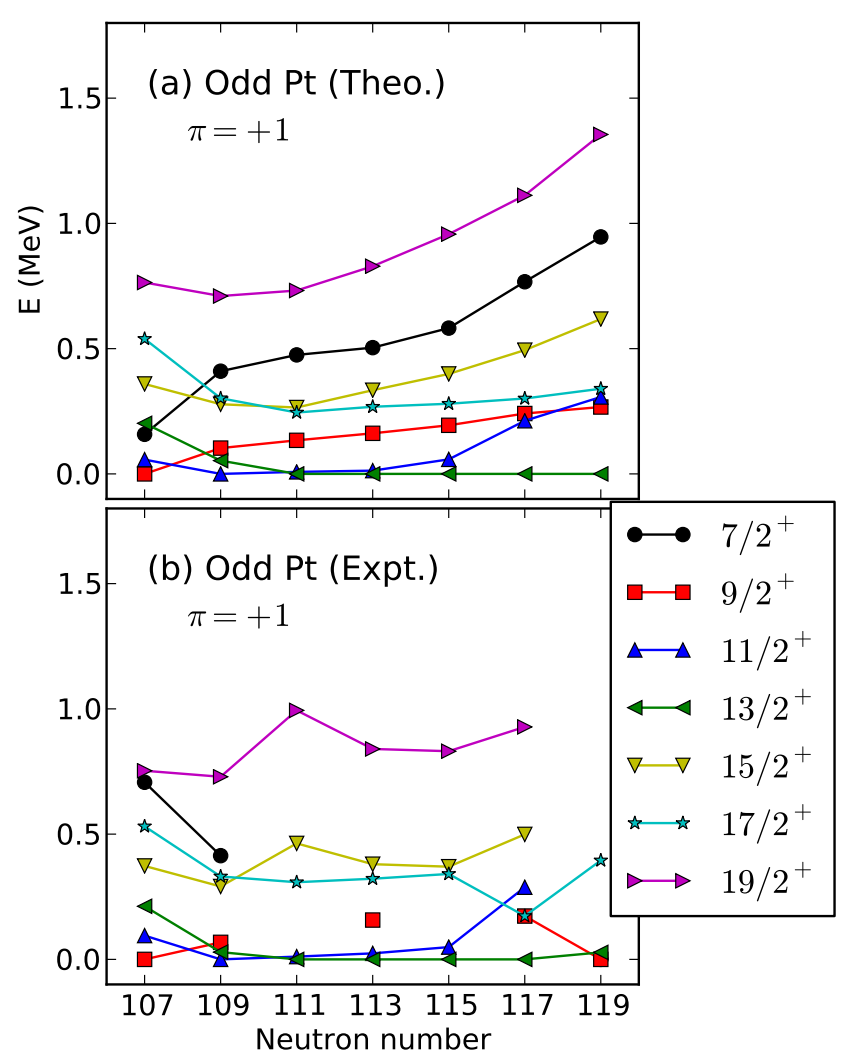

FIG. 8. (Color online) The same as in Fig. 7 but for the positive-parity states.

the Gogny-D1M energy surfaces (see, Fig. 1) suggesting a transition from a triaxial shape at ${ }^{192} \mathrm{Os}(N=116)$ to an oblate-soft one at ${ }^{194} \mathrm{Os}(N=118)$. At $N=109$ and 111 , the predicted ground-state spins for positive parity states (Fig. 10(a)) do not coincide with the experiment (Fig. 10(b)). This results from the fact, that the bosonfermion parameters for those nuclei have been chosen so as to reproduce the overall level structure up to the spin $J \sim 19 / 2^{+}$. However, we have also verified that if one attempts to reproduce the experimental ground-state's spin for ${ }^{185,187}$ Os, the whole spectrum becomes too compressed.

Similar observations apply to the results for the odd- $Z$ Ir nuclei, depicted in Figs. 11 and 12 For example, both the theoretical and experimental negative-parity spectra in Figs. 11(a) and 11(b), respectively, suggest a rapid structural change in going from $N=110$ to 112 . At those neutron numbers, the corresponding even-even $\mathrm{Pt}$ core nuclei undergo a structural change in their energy surfaces and spectroscopic properties (see, Figs. 1 and 6). 


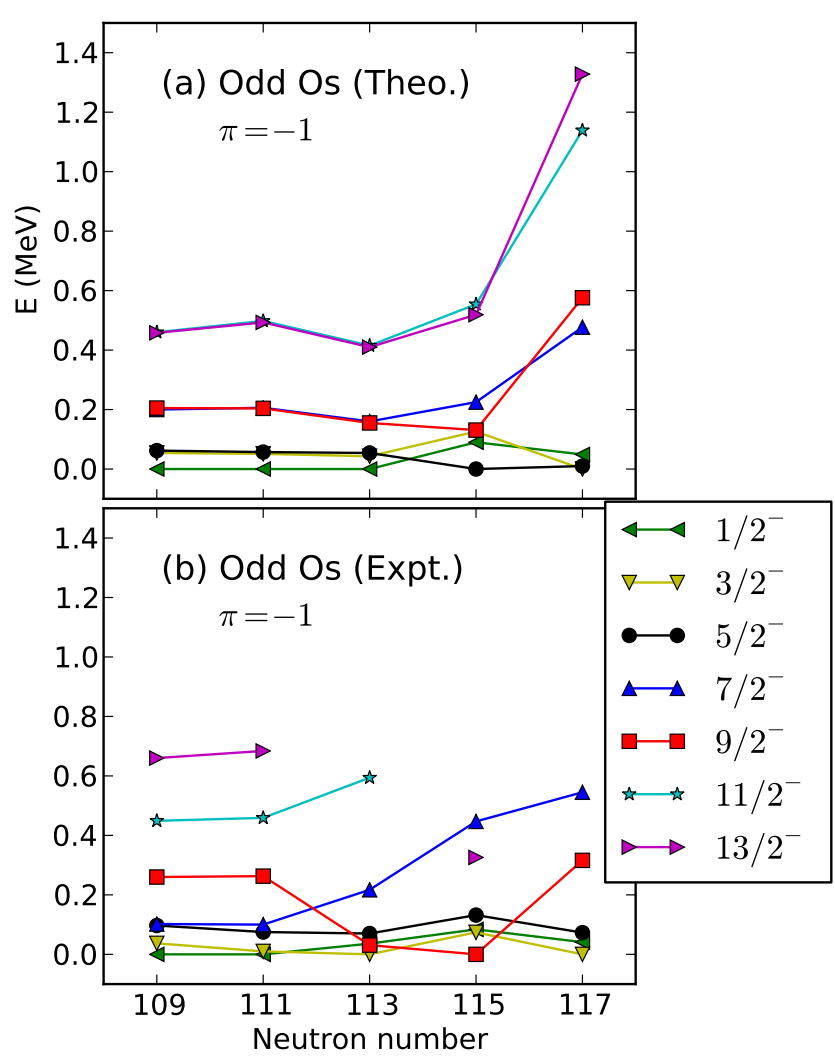

FIG. 9. (Color online) The same as in Fig. 7. but for the isotopes ${ }^{185-193}$ Os.

\section{Detailed level schemes for selected odd-mass nuclei}

In this section, we present a more detailed analysis of the nuclei ${ }^{195} \mathrm{Pt},{ }^{189} \mathrm{Os}$ and ${ }^{191} \mathrm{Ir}$, taken as illustrative examples. For them, abundant experimental information, especially for electromagnetic properties, is available for a detailed comparison with the theory predictions.

As can be seen from Fig. 13, our calculations reproduce reasonably well the experimental negative-parity yrast states for ${ }^{195} \mathrm{Pt}$. However, the predicted non-yrast levels tend to be overestimated like, for example, those experimental levels around $\approx 0.2 \mathrm{MeV}$ excitation energy. The discrepancies occur mainly because the single-particle energies and $v_{j}^{2}$ values used in the calculations may not be realistic enough to reproduce those levels. On the other hand, the agreement between the theoretical and experimental positive-parity levels is reasonable.

In Table I, we compare the predicted and experimental transition rates $B(E 2)$ and $B(M 1)$ as well as the spectroscopic quadrupole $Q_{J}$ and magnetic $\mu_{J}$ moments. The overall agreement is reasonably good. However, there are also some noticeable discrepancies. For instance, both the $B\left(E 2 ; 5 / 2_{2}^{-} \rightarrow 3 / 2_{1}^{-}\right)$and $B\left(M 1 ; 5 / 2_{2}^{-} \rightarrow 3 / 2_{1}^{-}\right)$values are significantly smaller than the experimental ones. The dominant components of the IBFM-2 wave function

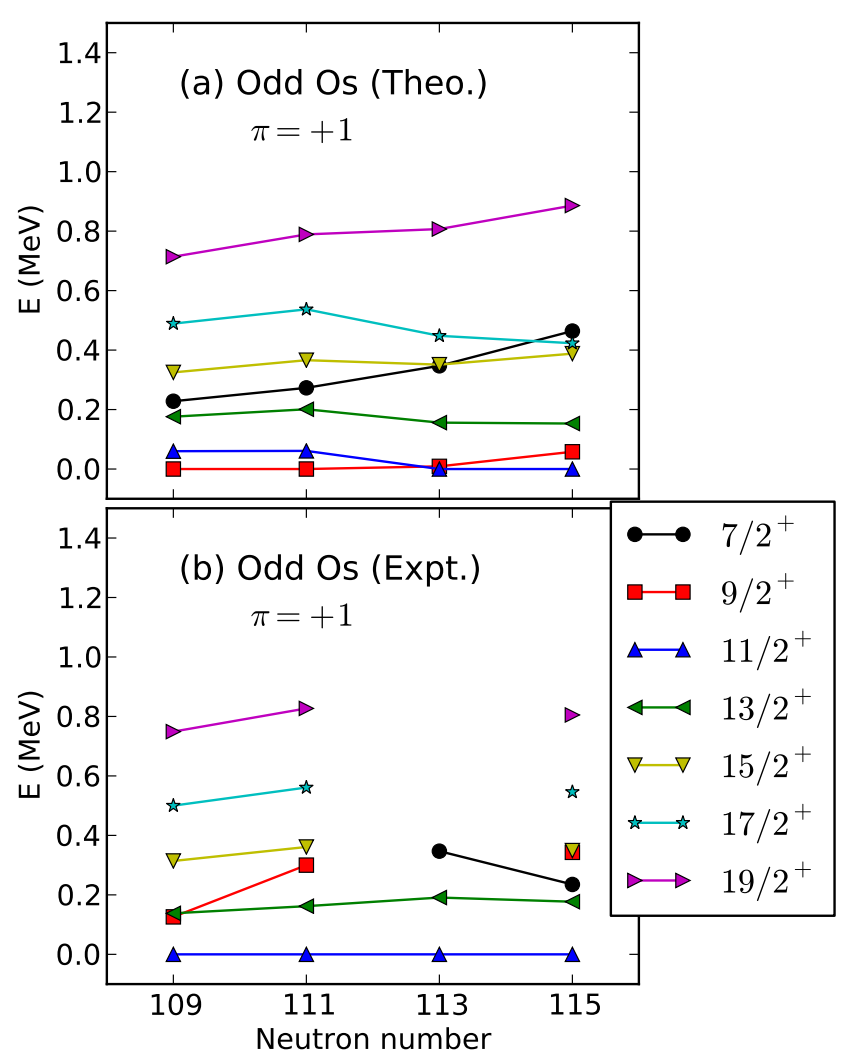

FIG. 10. (Color online) The same as in Fig. 8 but for the isotopes ${ }^{185-193}$ Os.

for the $5 / 2_{2}^{-}$state are $3 p_{3 / 2}(39 \%)$ and $2 f_{5 / 2}(45 \%)$, while those for the $3 / 2_{1}^{-}$state are $3 p_{1 / 2}(45 \%)$ and $3 p_{3 / 2}$ $(37 \%)$. Such a difference in the composition of the wave functions result in a small overlap between the two states that is the main responsible of the too small E2 and M1 transition rates predicted.

For ${ }^{189} \mathrm{Os}$, as seen in Fig. 14, the obtained level structure is similar to the one for ${ }^{195} \mathrm{Pt}$. However, the negative-parity spectrum differs from the experimental one, for example, with respect to the ground-state spin. In addition, the very low-lying $9 / 2_{1}^{-}$level near the ground state could not be reproduced. Empirically, the $9 / 2_{1}^{-}$ state arises from the $1 h_{9 / 2}$ orbital coming closer to the Fermi surface [36. However, in the calculations the single-particle energy for the $1 h_{9 / 2}$ orbital lies much higher than all the other orbitals (see, Fig. 4(c)). Let us remark, that such a feature cannot be controlled via three boson-fermion interaction strengths alone.

In Table II we compare the electromagnetic properties obtained for ${ }^{189}$ Os with the available experimental data [36, 37. Most of the discrepancy is found for those transitions that involve non-yrast states. Note that, indeed, the energy levels corresponding to those states are neither properly reproduced.

In Fig. 15 we compare the excitation spectra for ${ }^{191}$ Ir. 


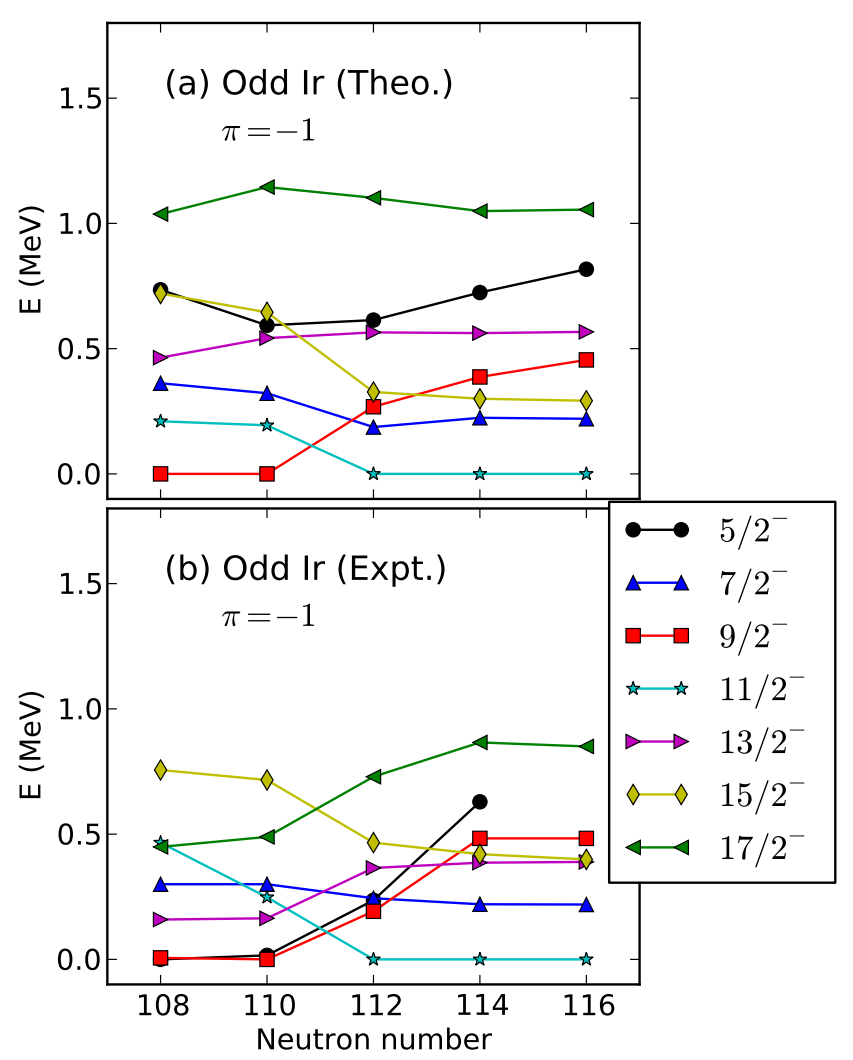

FIG. 11. (Color online) The same as in Fig. 7 but for the isotopes ${ }^{185-195} \mathrm{Ir}$.

Both the positive- and negative-parity states are rather well described. The electromagnetic properties computed for this nucleus are given in Table III. Although the corresponding energy levels are reasonably well described, some transition strengths, like the $B\left(E 2 ; 1 / 2_{1}^{+} \rightarrow 3 / 2_{1}^{+}\right)$ one, are significantly underestimated. The reason is that the IBFM-2 wave functions of the $3 / 2_{1}^{+}$and $1 / 2_{1}^{+}$states are mainly built from the $2 d_{3 / 2}(54 \%)$ and $3 s_{1 / 2}(58 \%)$ single-particle configurations, respectively. As a consequence, the E2 matrix element between the two states becomes too small. Keeping in mind that the employed model contains only three free parameters for each nucleus, the predicted electromagnetic properties in Table III for ${ }^{191} \mathrm{Ir}$, together with those for ${ }^{195} \mathrm{Pt}$ (Table I) and ${ }^{189}$ Os (Table II , appear to be rather reasonable.

\section{Signatures of shape phase transitions}

As yet another signature of the prolate-to-oblate shape phase transitions, we consider the quadrupole shape invariants [38] (denoted as q-invariants) $q_{m}(m=2,3, \ldots)$ obtained from the E2 matrix elements. These quantities have already been shown [17] to be good signatures of shape phase transitions involving $\gamma$-softness. For our

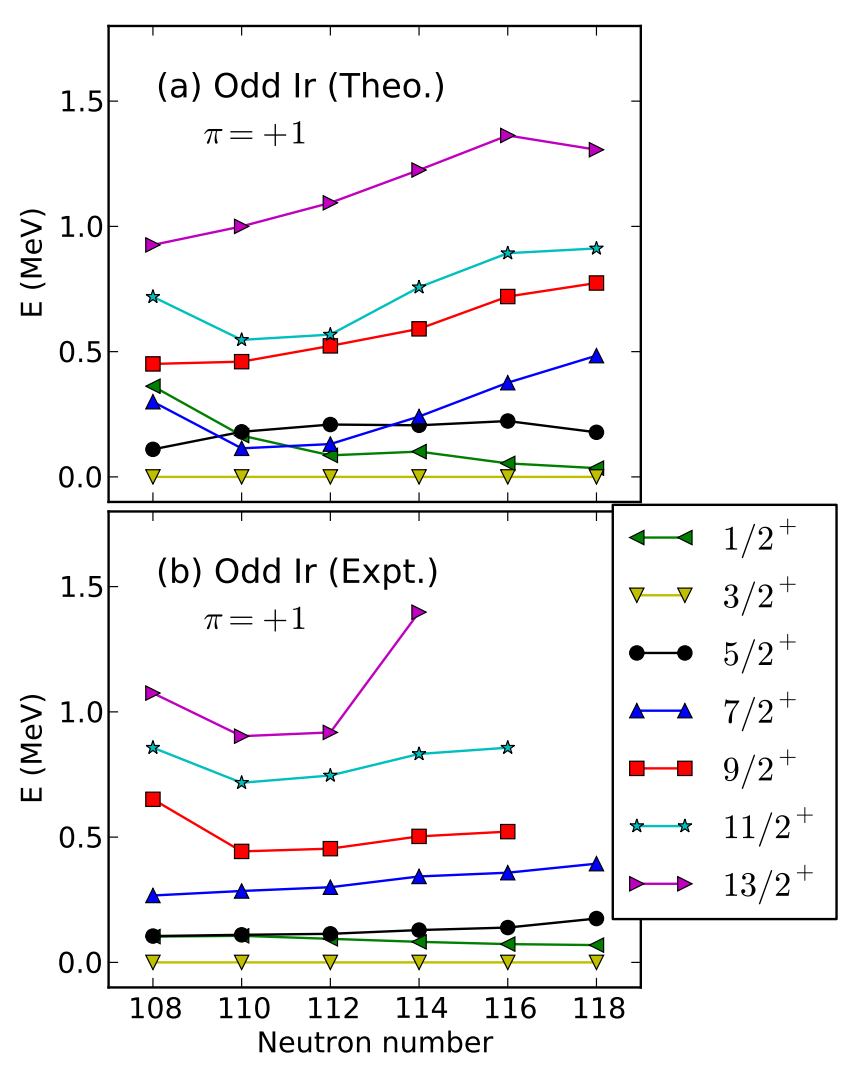

FIG. 12. (Color online) The same as in Fig. 8 but for the isotopes ${ }^{185-195} \mathrm{Ir}$.

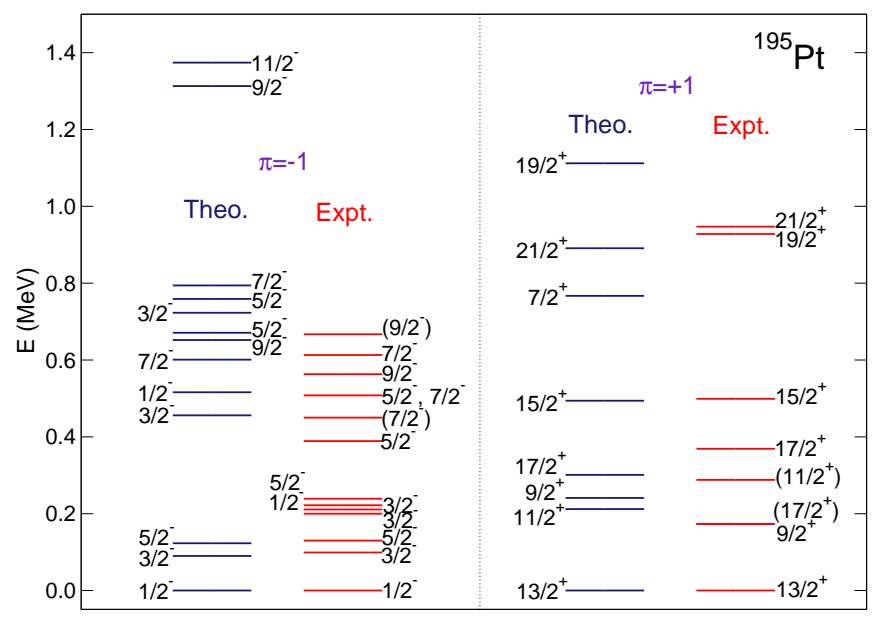

FIG. 13. (Color online) Comparison between the theoretical and experimental 36] low-lying positive- and negative-parity spectra for ${ }^{195} \mathrm{Pt}$.

purpose in this work, the relevant $q_{m}$ 's read

$$
q_{2}=\sum_{J^{\prime}} q_{2}\left(J^{\prime}\right)
$$




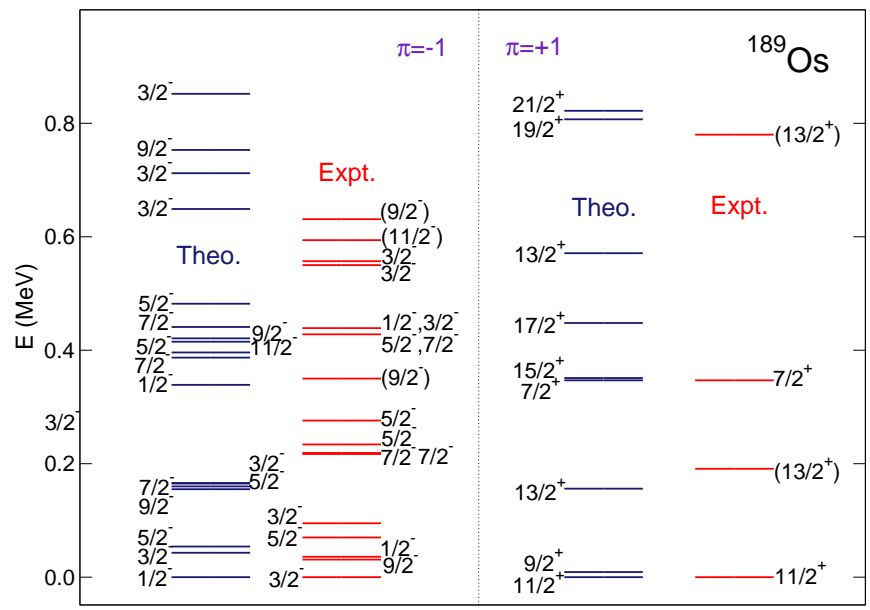

FIG. 14. (Color online) The same as in Fig. 13 but for ${ }^{189}$ Os.

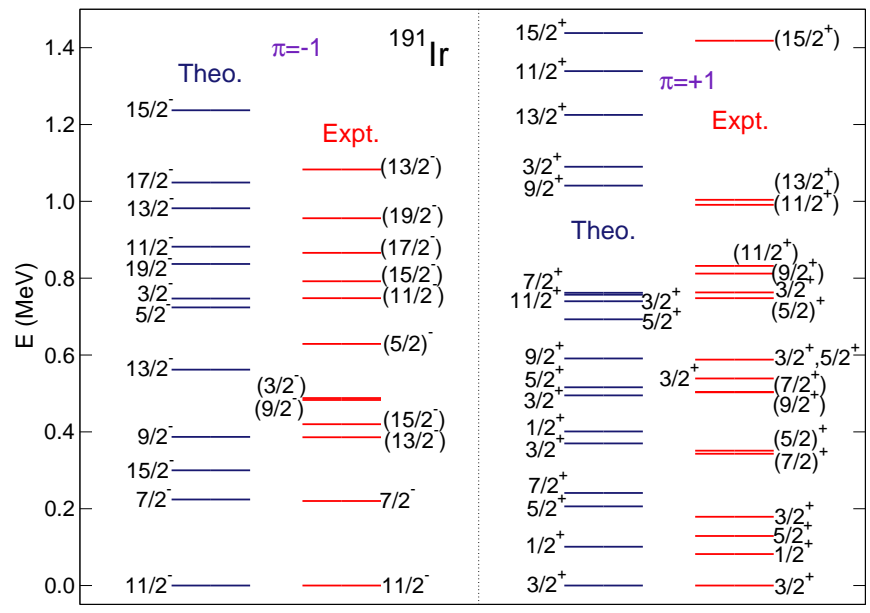

FIG. 15. (Color online) The same as in Fig. 13 but for ${ }^{191} \mathrm{Ir}$.

with

$$
q_{2}\left(J^{\prime}\right)=\sum_{i}^{n}\left\langle J\|\hat{Q}\| J_{i}^{\prime}\right\rangle\left\langle J_{i}^{\prime}\|\hat{Q}\| J\right\rangle
$$

and

$$
q_{3}=-\sqrt{\frac{7}{10}} \sum_{J^{\prime} J^{\prime \prime}} \sum_{i j}^{n}\left\langle J\|\hat{Q}\| J_{i}^{\prime}\right\rangle\left\langle J_{i}^{\prime}\|\hat{Q}\| J_{j}^{\prime \prime}\right\rangle\left\langle J_{j}^{\prime \prime}\|\hat{Q}\| J\right\rangle(7)
$$

In Eqs. (6) and (7), all possible E2 transition matrix elements among the states $J, J^{\prime}$ and $J^{\prime \prime}$, that satisfy the E2 selection rule, have been considered. The indices $i(j)$ in the sums are ordered according to increasing excitation energies of the $J^{\prime}\left(J^{\prime \prime}\right)$ levels and run up to $n=\infty$. However, we have confirmed that only a few of the lowest transitions contribute to the q-invariants significantly [17. In the case of even-even systems, the q-invariants for the $0_{1}^{+}$ground state (i.e, $J=0_{1}^{+}$and $J^{\prime}=2^{+}$) have been computed. The effective deformation parameters
TABLE I. The theoretical $B(E 2)$ and $B(M 1)$ transition probabilities (in Weisskopf units) and the $Q_{J}$ (in eb units) and $\mu_{J}$ (in $\mu_{N}$ units) values for ${ }^{195} \mathrm{Pt}$ are compared with the available

\begin{tabular}{|c|c|c|c|c|}
\hline & \multicolumn{2}{|c|}{$B(E 2)$ (W.u.) } & \multicolumn{2}{|c|}{$B(M 1)$ (W.u.) } \\
\hline & Th. & Exp. & Th. & Exp. \\
\hline $3 / 2_{1}^{-} \rightarrow 1 / 2_{1}^{-}$ & 24 & $11.5(15)$ & 0.034 & $0.0168(19)$ \\
\hline $3 / 2_{2}^{-} \rightarrow 1 / 2_{1}^{-}$ & 1.8 & $4.5(13)$ & 0.0066 & $0.00033(11)$ \\
\hline $3 / 2_{3}^{-} \rightarrow 1 / 2_{1}^{-}$ & 7.8 & $30(7)$ & 0.029 & $0.024(4)$ \\
\hline $3 / 2_{4}^{-} \rightarrow 1 / 2_{1}^{-}$ & 2.2 & $0.22(7)$ & 0.0060 & $0.0036(7)$ \\
\hline $5 / 2_{1}^{-} \rightarrow 1 / 2_{1}^{-}$ & 21 & $8.9(7)$ & - & - \\
\hline $5 / 2_{2}^{-} \rightarrow 1 / 2_{1}^{-}$ & 14 & $49(7)$ & - & - \\
\hline $5 / 2_{3}^{-} \rightarrow 1 / 2_{1}^{-}$ & 0.00043 & $1.3(9)$ & - & - \\
\hline $3 / 2_{4}^{-} \rightarrow 1 / 2_{2}^{-}$ & 2.2 & $<37$ & 0.0083 & $>0.00054$ \\
\hline $3 / 2_{2}^{-} \rightarrow 3 / 2_{1}^{-}$ & 15 & $0.05_{-5}^{+106}$ & 0.014 & $0.0030(8)$ \\
\hline $3 / 2_{4}^{-} \rightarrow 3 / 2_{1}^{-}$ & 4.1 & $0.07(6)$ & 0.027 & $0.0013(3)$ \\
\hline $5 / 2_{1}^{-} \rightarrow 3 / 2_{1}^{-}$ & 10 & $4.8(19)$ & 0.012 & $0.0269(21)$ \\
\hline $5 / 2_{2}^{-} \rightarrow 3 / 2_{1}^{-}$ & 0.082 & $11(6)$ & 0.0015 & $0.019(3)$ \\
\hline $5 / 2_{3}^{-} \rightarrow 3 / 2_{1}^{-}$ & 11 & $38(20)$ & 0.041 & $0.038(17)$ \\
\hline $5 / 2_{4}^{-} \rightarrow 3 / 2_{1}^{-}$ & - & - & 0.00050 & $<0.013$ \\
\hline $7 / 2_{2}^{-} \rightarrow 3 / 2_{1}^{-}$ & 4.1 & $29(10)$ & - & - \\
\hline $5 / 2_{4}^{-} \rightarrow 3 / 2_{3}^{-}$ & - & - & 0.0017 & $<0.017$ \\
\hline $7 / 2_{2}^{-} \rightarrow 3 / 2_{3}^{-}$ & 2.2 & $7(3)$ & - & - \\
\hline $7 / 2_{3}^{-} \rightarrow 3 / 2_{3}^{-}$ & 11 & $26(17)$ & - & - \\
\hline $5 / 2_{3}^{-} \rightarrow 5 / 2_{1}^{-}$ & 0.57 & 0.0097 & 0.0044 & $0.026(12)$ \\
\hline $7 / 2_{2}^{-} \rightarrow 5 / 2_{1}^{-}$ & - & - & 0.031 & $0.014(5)$ \\
\hline $9 / 2_{1}^{-} \rightarrow 5 / 2_{1}^{-}$ & 45 & $35(8)$ & - & - \\
\hline $5 / 2_{3}^{-} \rightarrow 5 / 2_{2}^{-}$ & - & - & 0.057 & $0.030(15)$ \\
\hline $5 / 2_{4}^{-} \rightarrow 5 / 2_{2}^{-}$ & 0.33 & $<60$ & - & - \\
\hline $7 / 2_{3}^{-} \rightarrow 5 / 2_{2}^{-}$ & 0.12 & $<210$ & 0.00077 & $<0.077$ \\
\hline $9 / 2_{2}^{-} \rightarrow 5 / 2_{2}^{-}$ & 34 & $30(8)$ & - & - \\
\hline \multirow[t]{3}{*}{$7 / 2_{2}^{-} \rightarrow 5 / 2_{3}^{-}$} & 0.11 & $<3.9 \times 10^{3}$ & 0.00042 & $<0.14$ \\
\hline & \multicolumn{2}{|c|}{$Q_{J}(e \mathrm{~b})$} & \multicolumn{2}{|c|}{$\mu_{J}\left(\mu_{N}\right)$} \\
\hline & Theo. & Exp. & Theo. & Exp. \\
\hline $1 / 2_{1}^{-}$ & - & - & +0.46 & $+0.60952(6)$ \\
\hline $3 / 2_{1}^{-}$ & +0.46 & & -0.37 & $-0.62(6)$ \\
\hline $3 / 2_{3}^{-}$ & +0.083 & & -0.59 & $+0.16(3)$ \\
\hline $5 / 2_{1}^{-}$ & +0.75 & & +0.90 & $+0.90(6)$ \\
\hline $5 / 2_{2}^{-}$ & +0.20 & & +1.06 & $+0.52(5)$ \\
\hline $5 / 2_{3}^{-}$ & +0.41 & & -0.062 & $+0.39(10)$ \\
\hline $5 / 2_{4}^{-}$ & -0.48 & & +0.87 & $+1.6(6)$ \\
\hline $7 / 2_{2}^{-}$ & +0.50 & & +0.84 & $+0.55(8)$ \\
\hline $7 / 2_{3}^{-}$ & +0.26 & & +0.78 & $+1.4(4)$ \\
\hline $7 / 2_{5}^{-}$ & +0.11 & & +0.76 & $+1.2(3)$ \\
\hline $9 / 2_{2}^{-}$ & +0.70 & & +1.53 & $+1.55(12)$ \\
\hline $9 / 2_{3}^{-}$ & +0.76 & & +0.59 & $+1.52(16)$ \\
\hline $13 / 2_{1}^{+}$ & +0.79 & $+1.4(6)$ & -1.31 & $-0.606(105)$ \\
\hline
\end{tabular}
experimental data [36, 37.

$\beta_{\text {eff }}$ and $\gamma_{\text {eff }}$ are obtained from the $q_{2}$ and $q_{3}$ values by the formulas

$$
\begin{aligned}
& \beta_{\mathrm{eff}}=\frac{4 \pi}{3 Z R_{0}^{2}} \sqrt{\sum_{J^{\prime}} \frac{1}{2 J^{\prime}+1}\left(J^{\prime} 2 J 0 \mid J J\right)^{-2} q_{2}\left(J^{\prime}\right)} \\
& \gamma_{\mathrm{eff}}=\frac{1}{3} \arccos \frac{q_{3}}{q_{2}^{3 / 2}}
\end{aligned}
$$


TABLE II. The same as in Table I but for ${ }^{189}$ Os.

\begin{tabular}{|c|c|c|c|c|}
\hline & \multicolumn{2}{|c|}{$B(E 2)$ (W.u.) } & \multicolumn{2}{|c|}{$B(M 1)$ (W.u.) } \\
\hline & Theo. & Exp. & Theo. & Exp. \\
\hline $3 / 2_{2}^{-} \rightarrow 1 / 2_{1}^{-}$ & 1.9 & $27(16)$ & 0.00044 & $0.048(8)$ \\
\hline $5 / 2_{1}^{-} \rightarrow 1 / 2_{1}^{-}$ & 61 & $24(3)$ & - & - \\
\hline $5 / 2_{2}^{-} \rightarrow 1 / 2_{1}^{-}$ & 4.8 & $25_{-8}^{+5}$ & - & - \\
\hline $5 / 2_{3}^{-} \rightarrow 1 / 2_{1}^{-}$ & 0.0029 & $0.6(4)$ & - & - \\
\hline $1 / 2_{1}^{-} \rightarrow 3 / 2_{1}^{-}$ & 134 & $27(7)$ & 0.0070 & $0.042(3)$ \\
\hline $3 / 2_{2}^{-} \rightarrow 3 / 2_{1}^{-}$ & 45 & $14(3)$ & 0.014 & $0.0032(6)$ \\
\hline $5 / 2_{1}^{-} \rightarrow 3 / 2_{1}^{-}$ & 7.7 & $100(10)$ & 0.019 & $0.0026(2)$ \\
\hline $5 / 2_{2}^{-} \rightarrow 3 / 2_{1}^{-}$ & 10 & $10_{-4}^{+3}$ & 0.00058 & $0.0005_{-4}^{+3}$ \\
\hline $5 / 2_{3}^{-} \rightarrow 3 / 2_{1}^{-}$ & 3.9 & $1.5(3)$ & 0.0021 & $8.9 \times 10^{-5}(23)$ \\
\hline $7 / 2_{1}^{-} \rightarrow 3 / 2_{1}^{-}$ & 82 & $18.2(11)$ & - & - \\
\hline $7 / 2_{2}^{-} \rightarrow 3 / 2_{1}^{-}$ & 0.94 & $38(2)$ & - & - \\
\hline $5 / 2_{2}^{-} \rightarrow 3 / 2_{2}^{-}$ & 9.0 & $17_{-7}^{+6}$ & 0.018 & $0.0012_{-5}^{+4}$ \\
\hline $5 / 2_{3}^{-} \rightarrow 3 / 2_{2}^{-}$ & 50 & $0.53(50)$ & 0.0013 & $<7.0 \times 10^{-5}$ \\
\hline $7 / 2_{1}^{-} \rightarrow 3 / 2_{2}^{-}$ & 0.00077 & $1.75(22)$ & - & - \\
\hline $7 / 2_{2}^{-} \rightarrow 3 / 2_{2}^{-}$ & 7.7 & $5(3)$ & - & - \\
\hline $3 / 2_{2}^{-} \rightarrow 5 / 2_{1}^{-}$ & 13 & $80_{-40}^{+90}$ & 0.00057 & $0.011(7)$ \\
\hline $5 / 2_{2}^{-} \rightarrow 5 / 2_{1}^{-}$ & 32 & $<16$ & 0.0018 & $0.00087(63)$ \\
\hline $5 / 2_{3}^{-} \rightarrow 5 / 2_{1}^{-}$ & 0.49 & $1.05(33)$ & 0.0097 & $<3.3 \times 10^{-5}$ \\
\hline $7 / 2_{1}^{-} \rightarrow 5 / 2_{1}^{-}$ & 10 & $14(6)$ & 0.00059 & $0.0008(4)$ \\
\hline $7 / 2_{2}^{-} \rightarrow 5 / 2_{1}^{-}$ & 7.0 & $43(2)$ & - & - \\
\hline $5 / 2_{3}^{-} \rightarrow 7 / 2_{2}^{-}$ & - & - & 0.029 & $0.0099(21)$ \\
\hline $5 / 2_{3}^{-} \rightarrow 9 / 2_{1}^{-}$ & 1.9 & $41(8)$ & - & - \\
\hline $7 / 2_{1}^{-} \rightarrow 9 / 2_{1}^{-}$ & 1.5 & $<2.2$ & 0.026 & $0.00107(17)$ \\
\hline \multirow[t]{3}{*}{$7 / 2_{2}^{-} \rightarrow 9 / 2_{1}^{-}$} & 18 & $6_{-1}^{+2}$ & 0.000 & $0.00025_{-14}^{+11}$ \\
\hline & \multicolumn{2}{|c|}{$Q_{J}(e \mathrm{~b})$} & \multicolumn{2}{|c|}{$\mu_{J}\left(\mu_{N}\right)$} \\
\hline & Theo. & Exp. & Theo. & Exp. \\
\hline $1 / 2_{1}^{-}$ & - & - & +0.45 & $+0.23(3)$ \\
\hline $3 / 2_{1}^{-}$ & -0.53 & $+0.98(6)$ & +0.17 & +0.6599 \\
\hline $5 / 2_{1}^{-}$ & -1.03 & $-0.63(2)$ & +0.96 & $+0.988(6)$ \\
\hline
\end{tabular}

where $R_{0}=1.2 A^{1 / 3} \mathrm{fm}$ and $\left(J^{\prime} 2 J 0 \mid J J\right)$ represents a Clebsch-Gordan coefficient.

In Fig. 16, we have depicted the $\beta_{\text {eff }}$ and $\gamma_{\text {eff }}$ values for the even-even $\mathrm{Pt}$ and Os nuclei. A monotonic decrease of $\beta_{\text {eff }}$ is observed in Fig. 16(a) as one approaches the $N=126$ shell closure. This agrees well with the gradual shift, from $\beta \approx 0.20$ to $\beta \approx 0$, in the global minima of the Gogny-D1M energy surfaces (see, Fig. 1). On the other hand, the $\gamma_{\text {eff }}$ value, plotted in Fig. 16(b), exhibits a faster change with $N$, jumping from $\gamma_{\mathrm{eff}} \approx 25^{\circ}$ (at $N=110$ ) to $\gamma_{\text {eff }} \approx 40^{\circ}$ (from $N=110$ onward) in $\mathrm{Pt}$ isotopes. Furthermore, the rate change is slower in the Os isotopes from $N=114$ up to 118 . This behavior of $\gamma_{\text {eff }}$ confirms that the prolate-to-oblate shape transition takes place. It is also consistent with the systematics of the Gogny-D1M energy surfaces.

Similar plots of $\beta_{\text {eff }}$ and $\gamma_{\text {eff }}$, are shown in Fig. 17 for several configurations close to the ground states of oddmass nuclei. As can be seen in Figs. 17(a) (odd- $N \mathrm{Pt}$ ), 17(c) (odd- $N$ Os) and 17(e) (odd- $Z$ Ir), the deformation $\beta_{\text {eff }}$ for each state typically shows a smooth behavior as a function of $N$. This correlates well with the results
TABLE III. The same as in TableI, but for ${ }^{191} \mathrm{Ir}$.

\begin{tabular}{|c|c|c|c|c|}
\hline & \multicolumn{2}{|c|}{$B(E 2)$ (W.u.) } & \multicolumn{2}{|c|}{$B(M 1)$ (W.u.) } \\
\hline & Theo. & Exp. & Theo. & Exp. \\
\hline $1 / 2_{2}^{+} \rightarrow 1 / 2_{1}^{+}$ & - & - & 0.00096 & $<0.0038$ \\
\hline $3 / 2_{2}^{+} \rightarrow 1 / 2_{1}^{+}$ & 2.4 & $58(9)$ & 0.00064 & $0.066(8)$ \\
\hline $3 / 2_{3}^{+} \rightarrow 1 / 2_{1}^{+}$ & 15 & $0.39(10)$ & 0.00016 & $0.00204(21)$ \\
\hline $5 / 2_{1}^{+} \rightarrow 1 / 2_{1}^{+}$ & 0.37 & $10.4(13)$ & - & - \\
\hline $5 / 2_{2}^{+} \rightarrow 1 / 2_{1}^{+}$ & 5.5 & $39(7)$ & - & - \\
\hline $1 / 2_{1}^{+} \rightarrow 3 / 2_{1}^{+}$ & 0.32 & $20.9(7)$ & $3.3 \times 10^{-6}$ & $0.000474(14)$ \\
\hline $1 / 2_{2}^{+} \rightarrow 3 / 2_{1}^{+}$ & 13 & $<2.7$ & 0.0070 & $<0.000277(23)$ \\
\hline $3 / 2_{2}^{+} \rightarrow 3 / 2_{1}^{+}$ & 24 & $15.7(20)$ & 0.029 & $0.00205(24)$ \\
\hline $3 / 2_{4}^{+} \rightarrow 3 / 2_{1}^{+}$ & 0.27 & $2.52(25)$ & $4.9 \times 10^{-5}$ & $0.0040(4)$ \\
\hline $3 / 2_{5}^{+} \rightarrow 3 / 2_{1}^{+}$ & 0.23 & - & $8.2 \times 10^{-5}$ & $\approx 0.17$ \\
\hline $5 / 2_{1}^{+} \rightarrow 3 / 2_{1}^{+}$ & 49 & $96.2(24)$ & 0.11 & $0.0259(6)$ \\
\hline $5 / 2_{2}^{+} \rightarrow 3 / 2_{1}^{+}$ & 9.6 & $1.7(4)$ & 0.044 & $0.0060(9)$ \\
\hline $5 / 2_{3}^{+} \rightarrow 3 / 2_{1}^{+}$ & 0.011 & - & 0.0087 & $\approx 0.17$ \\
\hline $7 / 2_{1}^{+} \rightarrow 3 / 2_{1}^{+}$ & 21 & $41.9(20)$ & - & - \\
\hline $7 / 2_{2}^{+} \rightarrow 3 / 2_{1}^{+}$ & 8.1 & $8.9(11)$ & - & - \\
\hline $3 / 2_{3}^{\overline{+}} \rightarrow 3 / 2_{2}^{+}$ & 7.2 & $0.013(10)$ & 0.067 & $0.0080(7)$ \\
\hline $5 / 2_{2}^{+} \rightarrow 3 / 2_{2}^{+}$ & 1.9 & $3.9(9)$ & 0.0015 & $0.056(8)$ \\
\hline $1 / 2_{2}^{+} \rightarrow 5 / 2_{1}^{+}$ & 3.4 & $<1.3$ & - & - \\
\hline $3 / 2_{2}^{+} \rightarrow 5 / 2_{1}^{+}$ & 24 & $27(9)$ & 0.00055 & $0.0053(11)$ \\
\hline $3 / 2_{3}^{+} \rightarrow 5 / 2_{1}^{+}$ & 4.7 & $0.60(9)$ & 0.00034 & $0.0071(8)$ \\
\hline $7 / 2_{1}^{+} \rightarrow 5 / 2_{1}^{+}$ & 6.8 & $29.8(15)$ & 0.0071 & $0.0296(15)$ \\
\hline $7 / 2_{2}^{+} \rightarrow 5 / 2_{1}^{+}$ & 13 & $18(3)$ & 0.022 & $0.0117(18)$ \\
\hline $9 / 2_{1}^{+} \rightarrow 5 / 2_{1}^{+}$ & 33 & $72(11)$ & - & - \\
\hline $3 / 2_{3}^{+} \rightarrow 5 / 2_{2}^{+}$ & 0.022 & $10(4)$ & 0.0052 & $0.0031(5)$ \\
\hline $11 / 2_{1}^{+} \rightarrow 7 / 2_{1}^{+}$ & 23 & $70(4)$ & - & - \\
\hline $3 / 2_{1}^{-} \rightarrow 7 / 2_{1}^{-}$ & 80 & $>38$ & - & - \\
\hline \multirow[t]{3}{*}{$7 / 2_{1}^{-} \rightarrow 11 / 2_{1}^{-}$} & 78 & $56(5)$ & - & - \\
\hline & \multicolumn{2}{|c|}{$Q_{J}(e \mathrm{~b})$} & \multicolumn{2}{|c|}{$\mu_{J}\left(\mu_{N}\right)$} \\
\hline & Theo. & Exp. & Theo. & Exp. \\
\hline $1 / 2_{1}^{+}$ & - & - & +1.20 & $+0.600(6)$ \\
\hline $3 / 2_{1}^{+}$ & +0.32 & $+0.816(9)$ & +0.29 & $+0.1507(6)$ \\
\hline $5 / 2_{1}^{+}$ & & - & +1.37 & $+0.81(6)$ \\
\hline $7 / 2_{1}^{+}$ & & - & +0.99 & $+1.40(6)$ \\
\hline $9 / 2_{1}^{+}$ & & - & +2.11 & $+2.4(2)$ \\
\hline $11 / 2_{1}^{-}$ & & - & +6.66 & $+6.03(4)$ \\
\hline
\end{tabular}

obtained for even-even systems (see, Fig. 16. On the other hand, as in the case of even-even systems, a rapid change of the $\gamma_{\text {eff }}$ value from below to above $\gamma_{\text {eff }} \approx 30^{\circ}$ occurs in some of the states and in each of the isotopic chains shown in panels (b), (d) and (f) of Fig. 17, i.e., in going from $N=109$ to 111 in odd- $N$ Pt (for the $3 / 2_{1}^{+}$ and $5 / 2_{1}^{+}$states), in going from $N=113$ to 117 in odd$N$ Os (for the $3 / 2_{1}^{+}$and $5 / 2_{1}^{+}$states) and in going from $N=110$ to 112 in odd- $Z$ Ir (for the $11 / 2_{1}^{-}$state). For those nuclei where $\gamma_{\text {eff }}$ changes abruptly, the associated even-even isotopes also show signs of a prolate-to-oblate shape transition (see, Fig. 16(b)). 


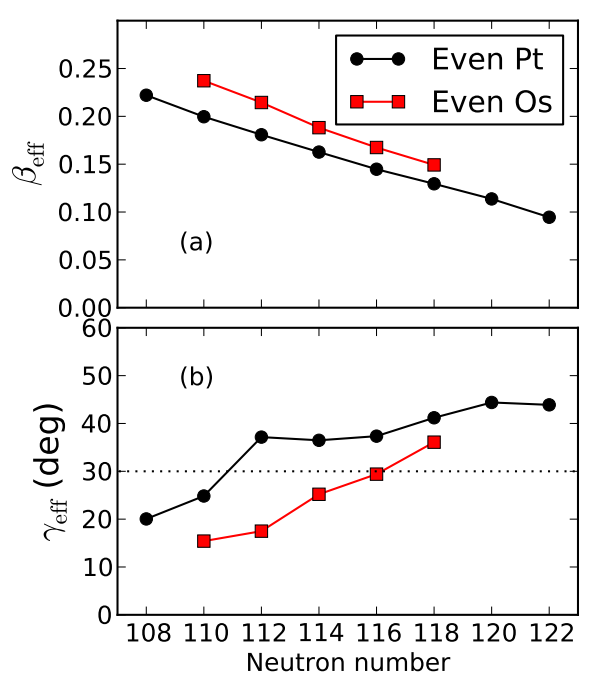

FIG. 16. (Color online) Effective $\beta$ and $\gamma$ deformation parameters for the even-even nuclei ${ }^{186-200} \mathrm{Pt}$ and ${ }^{186-194} \mathrm{Os}$ obtained from the E2 transition matrix elements.

\section{SUMMARY AND CONCLUDING REMARKS}

In this paper, we have studied the prolate-to-oblate shape phase transition in neutron-rich odd-mass nuclei with mass $A \approx 190$. Spectroscopic properties have been computed within a recently developed method where most of the parameters of the effective Hamiltonian of the IBFM are obtained from an EDF. To this end, the $(\beta, \gamma)$-deformation energy surfaces for the even-even core nuclei ${ }^{186-200} \mathrm{Pt}$ and ${ }^{186-194} \mathrm{Os}$, spherical single-particle energies and occupation probabilities for the corresponding odd-mass systems, have been computed within a microscopic EDF framework based on constrained mean field HFB configurations obtained with the Gogny-D1M parametrization. These quantities have then been used to determine the IBFM-2 Hamiltonian. The diagonalization of the IBFM-2 Hamiltonian allows to study the properties of ${ }^{185-195} \mathrm{Pt},{ }^{185-193} \mathrm{Os}$ and ${ }^{185-195} \mathrm{Ir}$. A few coupling constants, for the boson-fermion interaction, have been specifically fitted to the low-energy excitation spectra for each odd-mass nucleus. However, those parameters turned out to be almost constant or exhibit a gradual variation with nucleon number.

Our calculations account reasonably well for the spectroscopic properties of the studied odd-mass nuclei. In particular, we have identified a clear signature of a shape phase transitions by analyzing the systematic trend of the several calculated observables for the odd-mass nuclei. For instance, the evolution of the low-lying yrast states as well as the effective $\gamma$ deformation parameter exhibits significant structural changes at some specific neutron numbers. Our results point to the robustness of the prolate-to-oblate shape transitions in both even-even

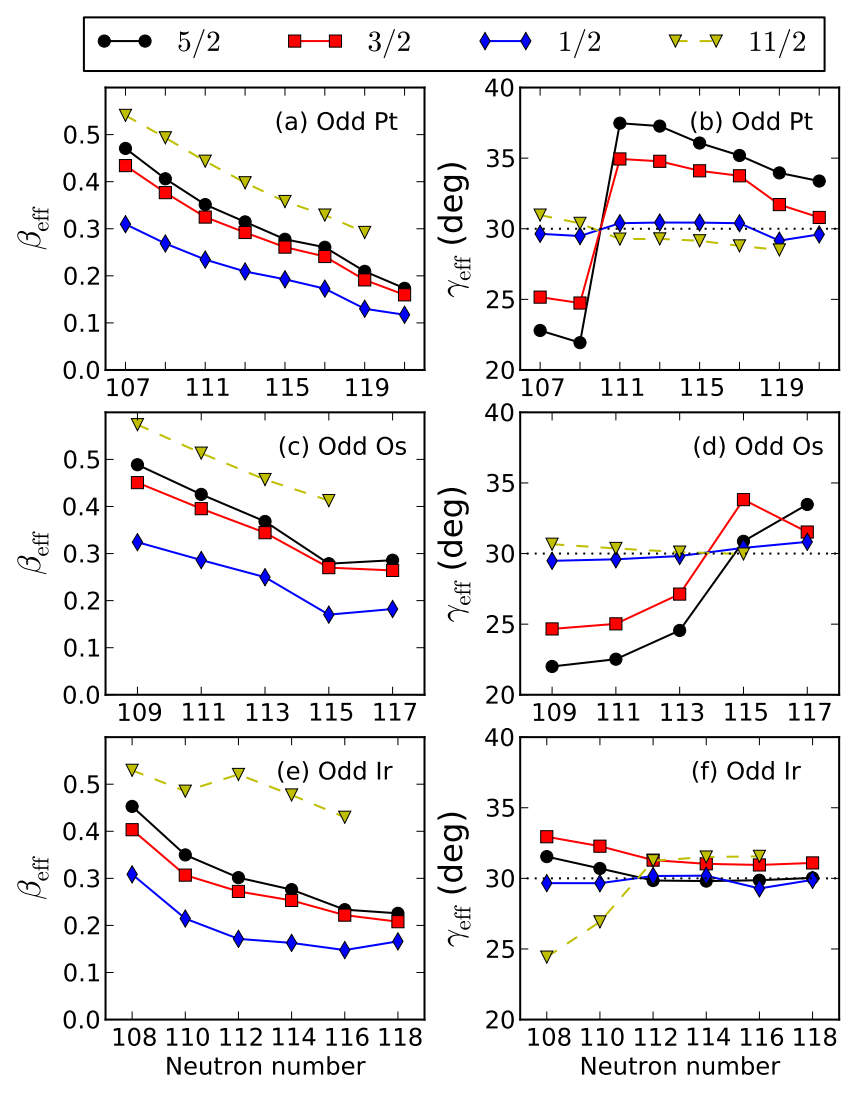

FIG. 17. (Color online) Effective $\beta$ and $\gamma$ deformation parameters for the odd- $N$ nuclei ${ }^{185-199} \mathrm{Pt}$ and ${ }^{185-193} \mathrm{Os}$ and the odd- $Z$ nuclei ${ }^{185-191}$ Ir for the $J^{\pi}=1 / 2_{1}^{\pi}, 3 / 2_{1}^{\pi}$ and $5 / 2_{1}^{\pi}$ states for normal-parity configurations $(\pi=-1$ for $\mathrm{Pt}$ and Os, and $\pi=+1$ for Ir) and $J^{\pi}=11 / 2_{1}^{\pi}$ state for uniqueparity configurations $(\pi=+1$ for $\mathrm{Pt}$ and Os, and $\pi=-1$ for Ir).

and odd-mass nuclei in this particular mass region. The present study could be extended further to another interesting case, such as those odd-mass nuclei in neutrondeficient $\mathrm{Pb}$ and $\mathrm{Hg}$ regions, which are characterized by a spectacular case of shape coexistence phenomena. This would require a major extension of the present method, and work along this line is in progress.

\section{ACKNOWLEDGMENTS}

This work was supported in part by the QuantiXLie Centre of Excellence, a project co-financed by the Croatian Government and European Union through the European Regional Development Fund - the Competitiveness and Cohesion Operational Programme (Grant KK.01.1.1.01.0004). The work of LMR was supported by Spanish Ministry of Economy and Competitiveness (MINECO) Grants No. FPA2015-65929-P and FIS201563770-P. 
[1] A. Bohr and B. M. Mottelsson, Nuclear Structure, vol. 2 (Benjamin, New York, USA, 1975).

[2] P. Cejnar, J. Jolie, and R. F. Casten, Rev. Mod. Phys. 82, 2155 (2010).

[3] T. Nikšić, D. Vretenar, G. A. Lalazissis, and P. Ring, Phys. Rev. Lett. 99, 092502 (2007).

[4] L. M. Robledo, R. Rodríguez-Guzmán, and P. Sarriguren, Journal of Physics G: Nuclear and Particle Physics 36, 115104 (2009), URL http://stacks.iop. org/0954-3899/36/i=11/a=115104.

[5] Z. P. Li, T. Nikšić, D. Vretenar, and J. Meng, Phys. Rev. C 81, 034316 (2010).

[6] N. Shimizu, T. Abe, M. Honma, T. Otsuka, T. Togashi, Y. Tsunoda, Y. Utsuno, and T. Yoshida, Physica Scripta 92, 063001 (2017), URL http://stacks .iop. org $/ 1402-4896 / 92 / i=6 / a=063001$.

[7] F. Iachello, Phys. Rev. Lett. 87, 052502 (2001).

[8] F. Iachello, Phys. Rev. Lett. 85, 3580 (2000), URL http: //link.aps.org/doi/10.1103/PhysRevLett.85.3580.

[9] J. Jolie, R. F. Casten, P. von Brentano, and V. Werner, Phys. Rev. Lett. 87, 162501 (2001), URL https://link. aps.org/doi/10.1103/PhysRevLett.87.162501.

[10] F. Iachello, A. Leviatan, and D. Petrellis, Phys. Lett. B 705, 379 (2011).

[11] A. Bohr, Mat. Fys. Medd. Dan. Vid. Selsk. 27, 16 (1953).

[12] K. Nomura, T. Nikšić, and D. Vretenar, Phys. Rev. C 93, 054305 (2016).

[13] F. Iachello and O. Scholten, Phys. Rev. Lett. 43, 679 (1979).

[14] F. Iachello and P. Van Isacker, The interacting bosonfermion model (Cambridge University Press, Cambridge, 1991).

[15] K. Nomura, T. Nikšić, and D. Vretenar, Phys. Rev. C 94, 064310 (2016), URL http://link.aps.org/doi/10. 1103/PhysRevC.94.064310.

[16] K. Nomura, R. Rodríguez-Guzmán, and L. M. Robledo, Phys. Rev. C 96, 014314 (2017), URL https://link. aps.org/doi/10.1103/PhysRevC.96.014314.

[17] K. Nomura, T. Nikšić, and D. Vretenar, Phys. Rev. C 96, 014304 (2017), URL https://link.aps.org/doi/ 10.1103/PhysRevC.96.014304.

[18] K. Nomura, R. Rodríguez-Guzmán, and L. M. Robledo, Phys. Rev. C 96, 064316 (2017), URL https://link. aps.org/doi/10.1103/PhysRevC.96.064316.

[19] K. Nomura, T. Nikšić, and D. Vretenar, Phys. Rev. C 97, 024317 (2018), URL https://link.aps.org/doi/
10.1103/PhysRevC.97.024317

[20] T. Otsuka, A. Arima, and F. Iachello, Nucl. Phys. A 309, 1 (1978).

[21] F. Iachello and A. Arima, The interacting boson model (Cambridge University Press, Cambridge, 1987).

[22] C. Alonso, J. Arias, R. Bijker, and F. Iachello, Physics Letters B 144, 141 (1984), ISSN 03702693, URL http://www.sciencedirect.com/science/ article/pii/0370269384917908

[23] S. Goriely, S. Hilaire, M. Girod, and S. Péru, Phys. Rev. Lett. 102, 242501 (2009).

[24] J. Decharge and M. Girod and D. Gogny, Phys. Lett. B 55, 361 (1975).

[25] J. M. Arias, C. E. Alonso, and M. Lozano, Phys. Rev. C 33, 1482 (1986), URL https://link.aps.org/doi/10. 1103/PhysRevC.33.1482

[26] K. Nomura, T. Otsuka, R. Rodríguez-Guzmán, L. M. Robledo, and P. Sarriguren, Phys. Rev. C 84, 054316 (2011).

[27] B. Bally, B. Avez, M. Bender, and P.-H. Heenen, Phys. Rev. Lett. 113, 162501 (2014).

[28] M. Borrajo and J. L. Egido, The European Physical Journal A 52, 277 (2016), ISSN 1434-601X, URL http: //dx.doi.org/10.1140/epja/i2016-16277-8.

[29] E. Caurier, G. Martínez-Pinedo, F. Nowacki, A. Poves, and A. P. Zuker, Rev. Mod. Phys. 77, 427 (2005).

[30] G. Colò, P. F. Bortignon, and G. Bocchi, Phys. Rev. C 95, 034303 (2017), URL https://link.aps.org/doi/ 10.1103/PhysRevC.95.034303

[31] K. Nomura, N. Shimizu, and T. Otsuka, Phys. Rev. Lett. 101, 142501 (2008).

[32] J. N. Ginocchio and M. W. Kirson, Nucl. Phys. A 350, $31(1980)$.

[33] K. Nomura, T. Otsuka, R. Rodríguez-Guzmán, L. M. Robledo, and P. Sarriguren, Phys. Rev. C 83, 014309 (2011).

[34] J. F. Berger, M. Girod, and D. Gogny, Nucl. Phys. A 428, 23 (1984).

[35] O. Scholten, Prog. Part. Nucl. Phys. 14, 189 (1985).

[36] Brookhaven National Nuclear Data Center, http://www.nndc.bnl.gov.

[37] N. Stone, At. Data Nucl. Data Tables 90, 75 (2005).

[38] D. Cline, Annual Review of Nuclear and Particle Science 36, 683 (1986), https://doi.org/10.1146/annurev.ns.36.120186.003343, URL https://doi.org/10.1146/annurev.ns.36. 120186.003343 